\title{
A Fractional-Order Discrete Noninvertible Map of Cubic Type: Dynamics, Control, and Synchronization
}

\author{
Xiaojun Liu $\mathbb{D}^{1},{ }^{1}$ Ling Hong $\mathbb{D},{ }^{2}$ Lixin Yang $\mathbb{D},{ }^{3}$ and Dafeng Tang ${ }^{4}$ \\ ${ }^{1}$ School of Sciences, Xi'an University of Posts and Telecommunications, Xi'an 710061, China \\ ${ }^{2}$ State Key Laboratory for Strength and Vibration of Mechanical Structures, Xi'an Jiaotong University, Xi'an 710049, China \\ ${ }^{3}$ School of Arts and Sciences, Shaanxi University of Science and Technology, Xi'an 710021, China \\ ${ }^{4}$ School of Automation, Xi'an University of Posts and Telecommunications, Xi'an 710061, China \\ Correspondence should be addressed to Xiaojun Liu; flybett3952@126.com
}

Received 6 December 2019; Revised 24 February 2020; Accepted 1 April 2020; Published 22 May 2020

Guest Editor: Viet-Thanh Pham

Copyright $\odot 2020$ Xiaojun Liu et al. This is an open access article distributed under the Creative Commons Attribution License, which permits unrestricted use, distribution, and reproduction in any medium, provided the original work is properly cited.

\begin{abstract}
In this paper, a new fractional-order discrete noninvertible map of cubic type is presented. Firstly, the stability of the equilibrium points for the map is examined. Secondly, the dynamics of the map with two different initial conditions is studied by numerical simulation when a parameter or a derivative order is varied. A series of attractors are displayed in various forms of periodic and chaotic ones. Furthermore, bifurcations with the simultaneous variation of both a parameter and the order are also analyzed in the three-dimensional space. Interior crises are found in the map as a parameter or an order varies. Thirdly, based on the stability theory of fractional-order discrete maps, a stabilization controller is proposed to control the chaos of the map and the asymptotic convergence of the state variables is determined. Finally, the synchronization between the proposed map and a fractional-order discrete Loren map is investigated. Numerical simulations are used to verify the effectiveness of the designed synchronization controllers.
\end{abstract}

\section{Introduction}

In the recent several decades, chaos is an attractive phenomenon in nonlinear dynamical systems, which has been extensively analyzed and studied deeply. It is well known that chaos was first detected in continuous nonlinear systems. Its characteristics and the existence in discrete dynamical maps have also been the interesting topics. Many discrete maps with chaotic attractors have been proposed, such as the Logistic map, Hénon map, and Lozi map [1-5]. With the rapid development of fractional calculus, many works including chaos, control, and synchronization for fractional-order continuous systems have been reported [6-18]. It should be pointed out that the fractional discrete maps were not paid enough attention and properly explored until recently [19].

In 1974, Diaz and Olser first put forward the fractional difference [20]. Up to now, fractional-order discrete maps have obtained more and more attention. In [21], a discrete fractional Hénon map was introduced, and its chaotic behavior was discussed. Dynamics, stabilization, and synchronization for several fractional-order maps, such as the Ikeda map, Loren map, and Lozi map, were studied in [22-28]. The discrete fractional calculus can avoid the tedious information or calculation error of the numerical discretization for the continuous ones due to the nonlocal property [29]. Therefore, more and more discrete maps with fractional operators need to be presented, and more abundant and complex dynamics behaviors need to be explored. Besides, it is well known that fractional-order discrete maps are sensitive not only to the small disturbance of parameters and initial conditions but also to the variation of fractional orders [30], which are the unique advantages of fractional-order systems. For this reason, a fractional-order discrete map is more suitable for data encryption and secure communications. Furthermore, fractional-order discrete maps have simple forms and rich dynamics, which are good for system analysis and numerical computation. Based on 
these, investigation of a new fractional-order discrete map including dynamics, stabilization, and synchronization is necessary and important for the development of fractional calculus.

In [31, 32], a two-dimensional noninvertible map with cubic order nonlinearity, which was taken as a chaotic cryptosystem, was proposed and studied. The evolution of attractors and their basins have been analyzed deeply and explained thoroughly. A noncyclic chaotic attractor for the map was displayed in [33]. Based on these, we extend the map to the fractional case and study its dynamics. The stability of the equilibrium points for the map is examined. By the bifurcation graphs and phase diagrams, the dynamics of the fractional-order discrete map with two different initial conditions is displayed as a parameter or a derivative order varies. Furthermore, bifurcations with the simultaneous variation of both a parameter and the order are also analyzed in the three-dimensional space. Interior crises occur in the map with the variation of a parameter or the order.

The main motivation of our work is to know whether bifurcations and chaos, which the integer-order discrete map possesses, also exist in the fractional-order counterpart. In fact, these dynamics behaviors do exist in the fractional-order map, and multifaceted complex dynamics is observed by means of the numerical simulations. For a chaotic system, control and synchronization are very important for its application in practical problems. In our work, we are also interested in studying the control and synchronization for the fractional-order map. Based on the stability theory of fractional-order discrete maps, a stabilization controller is proposed to control the chaos of the map. The synchronization between the proposed map and a fractional-order discrete Loren map is studied and realized.

\section{Discrete Fractional Calculus}

In this section, we will recall the definition and related theories for the discrete fractional calculus. In the following, the symbol ${ }^{C} \Delta_{a}^{v} X(t)$ represents the $v$ order Caputo type delta fractional calculus of a function $X(t): N_{a} \longrightarrow R$ with $N_{a}=$ $\{a, a+1, a+2, \ldots\}[34]$, which is expressed as follows:

$$
\begin{gathered}
{ }^{C} \Delta_{a}^{v} X(t)=\Delta_{a}^{-(n-v)} X(t)=\frac{1}{\Gamma(n-v)} \sum_{s=a}^{t-(n-v)} \\
\cdot(t-s-1)^{(n-v-1)} \Delta^{n} X(s),
\end{gathered}
$$

where $v \notin N$ is the order, $t \in N_{a+n-v}$, and $n=\lceil v\rceil+1$. In formula (1), the $v$ th fractional sum of $\Delta_{s}^{n} X(t)$ is defined as $[35,36]$

$$
\Delta_{a}^{-v} X(t)=\frac{1}{\Gamma(v)} \sum_{s=a}^{t-v}(t-s-1)^{(v-1)} X(s)
$$

where $t \in N_{a+v}, v>0 . t^{(v)}$ represents the falling function which is defined according to the Gamma function $\Gamma$ as

$$
t^{(v)}=\frac{\Gamma(t+1)}{\Gamma(t+1-v)} .
$$

Generally speaking, the following method is employed to compute the numerical solutions for a fractional-order discrete map.

For an equation with the fractional calculus operator,

$$
\left\{\begin{array}{l}
{ }^{C} \Delta_{a}^{v} u(t)=f(t+v-1, u(t+v-1)), \\
\Delta^{k} u(a)=u_{k} \cdot n=\lceil v\rceil+1, \quad k=0,1,2, \ldots, n-1,
\end{array}\right.
$$

the equivalent discrete integral one is

$$
\begin{aligned}
u(t)= & u_{0}(t)+\frac{1}{\Gamma(v)} \sum_{s=a+n-v}^{t-v}(t-s-1)^{(v-1)} f(s+v-1, u \\
& \cdot(s+v-1)), \quad t \in N_{a+n},
\end{aligned}
$$

where $u_{0}(t)=\sum_{k=0}^{n-1}\left((t-a)^{(k)} / \Gamma(k+1)\right) \Delta^{k} u(a)$.

The following theorem is used to analyze the stabilization and synchronization for fractional discrete maps. For the proof of the theorem, please refer to the literature [37].

Theorem 1. The zero equilibrium of a linear fractional discrete system:

$$
{ }^{C} \Delta_{a}^{v} X(t)=\mathbf{M} X(t+v-1),
$$

where $X(t)=\left(x_{1}(t), x_{2}(t), \cdots, x_{n}(t)\right)^{T}, 0<v \leq 1, \mathbf{M} \in R^{n \times n}$ and $\forall t \in N_{a+1-v}$, is asymptotically stable if

$$
\begin{array}{r}
\left|\lambda_{i}\right|<\left(2 \cos \frac{\left|\arg \lambda_{i}\right|-\pi}{2-v}\right)^{v}, \\
\left|\arg \lambda_{i}\right|>\frac{v \pi}{2}, \quad i=1,2, \ldots, n,
\end{array}
$$

for all the eigenvalues $\lambda$ of $\mathbf{M}$.

\section{A Fractional-Order Discrete Map}

3.1. Description of the Map. Firstly, the two-dimensional discrete map with cubic nonlinearity in [31-33] is described as follows:

$$
\left\{\begin{array}{l}
x(n+1)=y(n), \\
y(n+1)=b\left(-x^{3}(n)+x(n)\right)+c\left(-y^{3}(n)+y(n)\right),
\end{array}\right.
$$

where $x(n)$ and $y(n)$ are the state variables and $b$ and $c$ parameters. The first-order difference of (8) is formulated as

$$
\left\{\begin{array}{l}
x(n+1)=y(n)-x(n), \\
y(n+1)=b\left(-x^{3}(n)+x(n)\right)+c\left(-y^{3}(n)+y(n)\right)-y(n) .
\end{array}\right.
$$

By employing the Caputo-like delta difference given in (1) with the starting point $a$, the corresponding fractional map is 


$$
\left\{\begin{array}{l}
{ }^{C} \Delta_{a}^{v} x(t)=y(t-1+v)-x(t-1+v), \\
{ }^{C} \Delta_{a}^{v} y(t)=b\left(-x^{3}(t-1+v)+x(t-1+v)\right)+c\left(-y^{3}(t-1+v)+y(t-1+v)\right)-y(t-1+v) .
\end{array}\right.
$$

Based on equations (4) and (5), we can obtain

$$
\left\{\begin{array}{l}
x(t)=x(a)+\frac{1}{\Gamma(v)} \sum_{s=a+1}^{t-v}(t-s-1)^{(v-1)}(y(t-1+v)-x(t-1+v)), \\
y(t)=y(a)+\frac{1}{\Gamma(v)} \sum_{s=a+1}^{t-v}(t-s-1)^{(v-1)}\left(b\left(-x(t-1+v)^{3}+x(t-1+v)\right)\right. \\
\left.+c\left(-y(t-1+v)^{3}+y(t-1+v)\right)-y(t-1+v)\right),
\end{array}\right.
$$

where $(t-s-1)^{(v-1)} / \Gamma(v)$ is the discrete kernel function and $\quad\left((t-s-1)^{(v-1)} / \Gamma(v)\right)=(\Gamma(t-s) /(\Gamma(v) \Gamma(t-s-v+$
1))). Based on this, the numerical solution for the fractional discrete map (10) can be obtained, which is as follows:

$$
\left\{\begin{array}{l}
x(n)=x(a)+\frac{1}{\Gamma(v)} \sum_{j=1}^{n} \frac{\Gamma(n-j+v)}{\Gamma(n-j+1)}(y(j-1)-x(j-1)), \\
y(n)=y(a)+\frac{1}{\Gamma(v)} \sum_{j=1}^{n} \frac{\Gamma(n-j+v)}{\Gamma(n-j+1)}\left(b\left(-x^{3}(j-1)+x(j-1)\right)+c\left(-y^{3}(j-1)+y(j-1)\right)-y(j-1)\right) .
\end{array}\right.
$$

In the rest of the paper, the low limit $a$ is fixed as 0 .

3.2. Stability of Equilibrium Points. Now, we turn to study the stability of equilibrium points for map (10). By the simple computation, we can get the three equilibrium points:

$$
\begin{aligned}
& E_{1}(0,0), \\
& E_{2,3}\left( \pm \sqrt{1-\frac{1}{b+c}}, \pm \sqrt{1-\frac{1}{b+c}}\right),
\end{aligned}
$$

when $b+c>1$. The map has only one equilibrium point $E_{1}(0,0)$ when $b+c \leq 1$. The Jacobian matrix of map (10) evaluated at an equilibrium point $E_{*}=\left(x^{*}, y^{*}\right)$ is

$$
J_{1}=\left[\begin{array}{cc}
-1 & 1 \\
b\left(-3 x^{*}+1\right) & c\left(-3 y^{*}+1\right)-1
\end{array}\right] .
$$

The corresponding eigenvalues for the equilibrium point $E_{1}(0,0)$ are $\lambda_{1,2}=(c / 2) \pm\left(\sqrt{4 b+c^{2}} / 2\right)$. In this paper, we only consider the case of map (10) with positive parameters.
Therefore, the zero equilibrium point $E_{1}$ is unstable due to $\left|\arg \lambda_{1}\right|=0<(v \pi / 2)$ on the basis of Theorem 1 .

For a fractional-order discrete map, the stability of a zero equilibrium point can be determined easily based on Theorem 1 . Therefore, we will use a very simple method proposed in [38] for handling with the nonzero equilibrium points. For more special details about the method, please refer to Remark 2.5 in the literature [38].

In order to analyze the stability of the nonzero equilibrium points $E_{2,3}$, let $x_{2}=\sqrt{1-(1 /(b+c))}$, $y_{2}=\sqrt{1-(1 /(b+c))}, \quad x_{3}=-\sqrt{1-(1 /(b+c))}$, and $y_{3}=$ $-\sqrt{1-(1 /(b+c))}$. Through the following variable transforms,

$$
\left\{\begin{array}{l}
z_{21}(t-1+v)=x(t-1+v)-x_{2}, \\
z_{22}(t-1+v)=y(t-1+v)-y_{2}, \\
z_{31}(t-1+v)=x(t-1+v)-x_{3}, \\
z_{32}(t-1+v)=y(t-1+v)-y_{3},
\end{array}\right.
$$

we can get two newly maps with a zero equilibrium point: 


$$
\begin{aligned}
& \left\{\begin{array}{l}
{ }^{C} \Delta_{a}^{v}\left(z_{21}(t)+x_{2}\right)={ }^{C} \Delta_{a}^{v} z_{21}(t)=z_{22}(t-1+v)+y_{2}-z_{21}(t-1+v)-x_{2}, \\
{ }^{C} \Delta_{a}^{v}\left(z_{22}(t)+y_{2}\right)={ }^{C} \Delta_{a}^{v} z_{22}(t)=b\left(-\left(z_{21}(t-1+v)+x_{2}\right)^{3}+z_{21}(t-1+v)+x_{2}\right) \\
+c\left(-\left(z_{22}(t-1+v)+y_{2}\right)^{3}+z_{22}(t-1+v)+y_{2}\right)-z_{22}(t-1+v)-y_{2},
\end{array}\right. \\
& \left\{\begin{array}{l}
{ }^{C} \Delta_{a}^{v}\left(z_{31}(t)+x_{3}\right)={ }^{C} \Delta_{a}^{v} z_{31}(t)=z_{32}(t-1+v)+y_{3}-z_{31}(t-1+v)-x_{3}, \\
{ }^{C} \Delta_{a}^{v}\left(z_{32}(t)+y_{3}\right)={ }^{C} \Delta_{a}^{v} z_{32}(t)=b\left(-\left(z_{31}(t-1+v)+x_{3}\right)^{3}+z_{31}(t-1+v)+x_{3}\right) \\
+c\left(-\left(z_{32}(t-1+v)+y_{3}\right)^{3}+z_{32}(t-1+v)+y_{3}\right)-z_{32}(t-1+v)-y_{3},
\end{array}\right.
\end{aligned}
$$

which are corresponding to $E_{2,3}$, respectively. The Jacobian matrix of maps (16) and (17) evaluated at the zero equilibrium point is

$$
\begin{aligned}
J_{2} & =\left[\begin{array}{cc}
-1 & 1 \\
-3 b x_{2}^{2}+b & -3 c y_{2}^{2}+c-1
\end{array}\right] \\
& =\left[\begin{array}{cc}
-1 & 1 \\
-3 b x_{3}^{2}+b & -3 c y_{3}^{2}+c-1
\end{array}\right] .
\end{aligned}
$$

The corresponding eigenvalues of $J_{2}$ for the zero equilibrium point are

$$
\lambda_{3,4}=-\frac{2 b-c \pm \sqrt{-(2 b+2 c-3)\left(4 b^{2}-2 b c^{2}+4 b c-2 c^{3}+3 c^{2}\right)}+2 b c+2 c^{2}}{2(b+c)} .
$$

When the parameters of map (10) are choosen as $b=$ 2.2 and $c=0.95$ and the order is taken as $v=0.98$, the corresponding eigenvalues are $\lambda_{3,4}=-1.4976 \pm 1.4343 i$. By simple computation, we can obtain

$$
\begin{aligned}
\left|\arg \lambda_{i}\right| & =2.3788>\frac{v \pi}{2}=1.5379, \quad i=3,4, \\
\left|\lambda_{i}\right| & =2.0736>\left(2 \cos \frac{\left|\arg \lambda_{i}\right|-\pi}{2-v}\right)^{v}=1.4538, \quad i=3,4,
\end{aligned}
$$

which means the equilibrium point $E_{2,3}$ is unstable in this case according to Theorem 1.

\section{Dynamics Analysis}

In this section, the dynamics of the fractional-order discrete map (10) with the variation of a parameter or the fractional order and the bifurcations with the simultaneous variation of both a parameter and the order $v$ will be analyzed in detail.

4.1. Dynamics as the Parameter $b$ Varies. When the order $v=0.98$ and parameter $c=0.95$, the dynamics of map (10) with the variation of the parameter $b$ is analyzed. The bifurcation diagrams and the corresponding largest Lyapunov exponents (LLE) spectrums with two different initial conditions $x_{01}=(0.8,-0.4)$ and $x_{02}=(0.8,0.4)$ are displayed in Figure 1 . From which we can see that the dynamics of the map is abundant and shows a symmetry with different initial conditions in this case.

The evolution of the trajectories for different $b$ with $x_{01}$ is depicted in Figure 2. In the phase plane, there is a fixed point which means the map is period-1 for $b=1$ (Figures 2(a) and 2(b)). The map has a limit cycle attractor for $b=1.5$, see Figure 2(c), which means a Hopf bifurcation occurs as the parameter $b$ increases from 1 to 1.5 . The shape of the limit cycle changes as $b$ increases further (Figures 2(d) and 2(e)). The map keeps chaotic when $b$ varies from 1.75 to 2.2 . In Figure 2(g), three small chaotic attractors appear in the phase plane when $b=1.9$ and convert to one large attractor when $b=1.95$ (Figure 2(h)). From Figures 2(i) and 2(j), we can see that the chaotic attractor becomes a large one suddenly, which implies that an interior crisis occurs as $b$ increases from 2 to 2.2 .

The dynamics of the map with the initial condition $x_{02}$, which is similar to Figure 2, is displayed in Figure 3. From the global dynamics perspective, the two chaotic attractors, which are depicted in Figures 2(i) and 3(i), collide with each other and convert to a large one (Figures $2(\mathrm{j})$ and $3(\mathrm{j}))$.

4.2. Dynamics as the Parameterc Varies. The fractional order $v$ is fixed as 0.98 and the parameter $b=2.2$, and the dynamics of map (10) when $c$ is varied in the interval of $[0.2,1]$ with two initial conditions $x_{01}$ and $x_{02}$ are studied. Firstly, the bifurcation diagrams and the corresponding LLE spectrums with $x_{01}$ and $x_{02}$ are plotted in Figure 4. From 


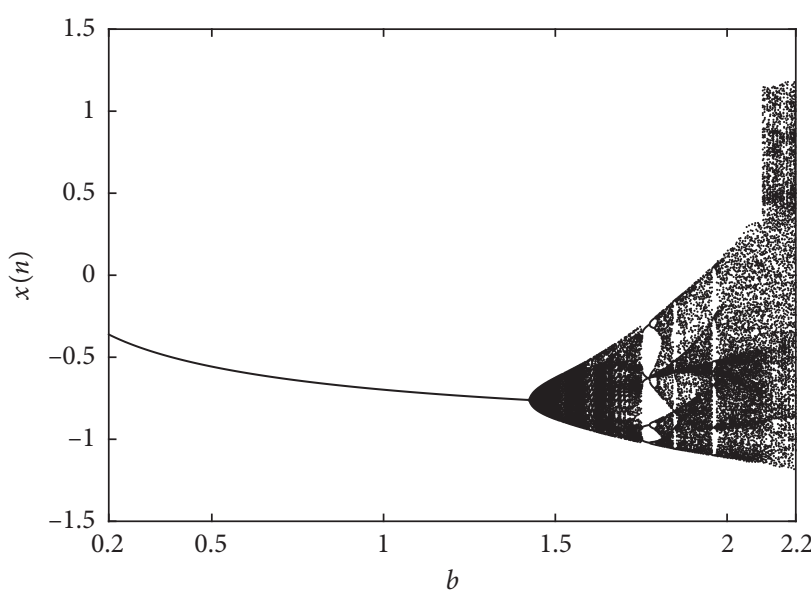

(a)

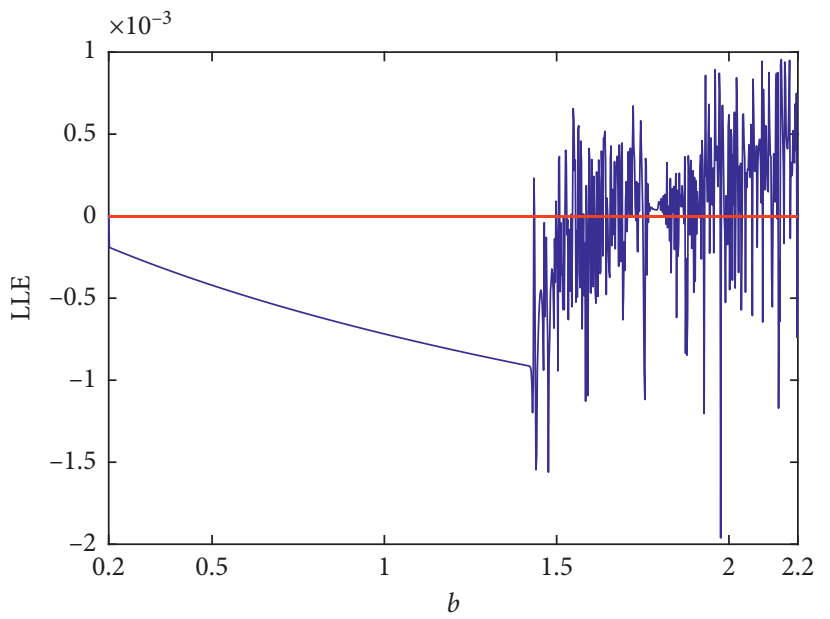

(c)

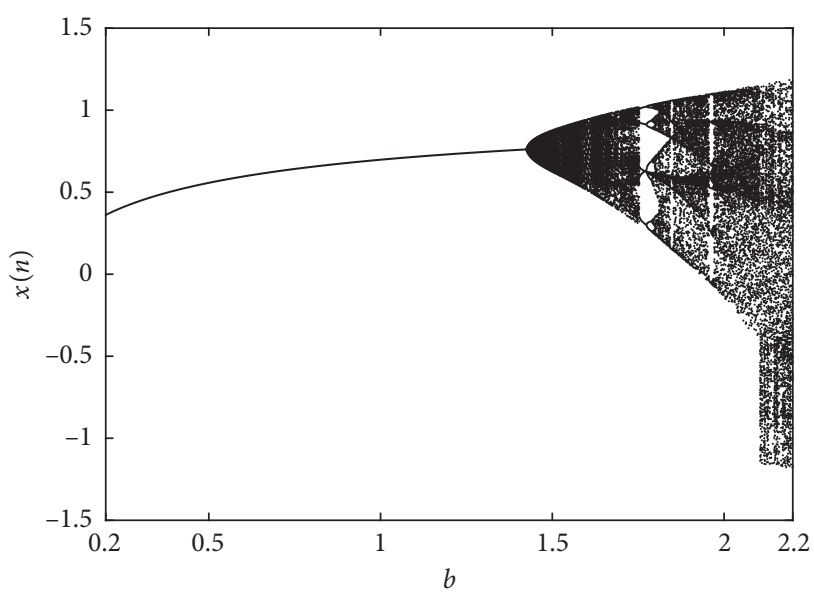

(b)

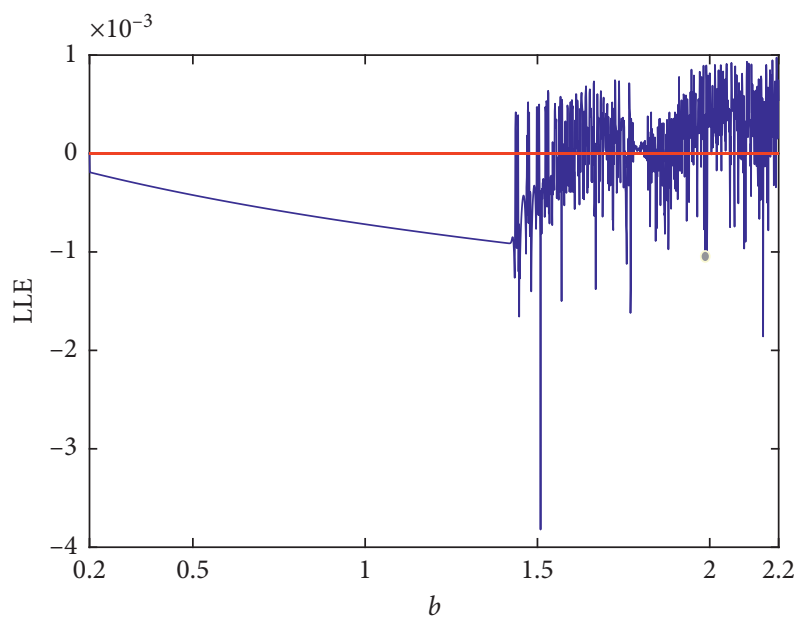

(d)

FIGURE 1: Bifurcation diagrams and corresponding LLE spectrums for the map with different initial values as the parameter $b$ varies: (a) the bifurcation diagram with $x_{01}$, (b) the bifurcation diagram with $x_{02}$, (c) the LLE spectrum with $x_{01}$, and (d) the LLE spectrum with $x_{02}$.

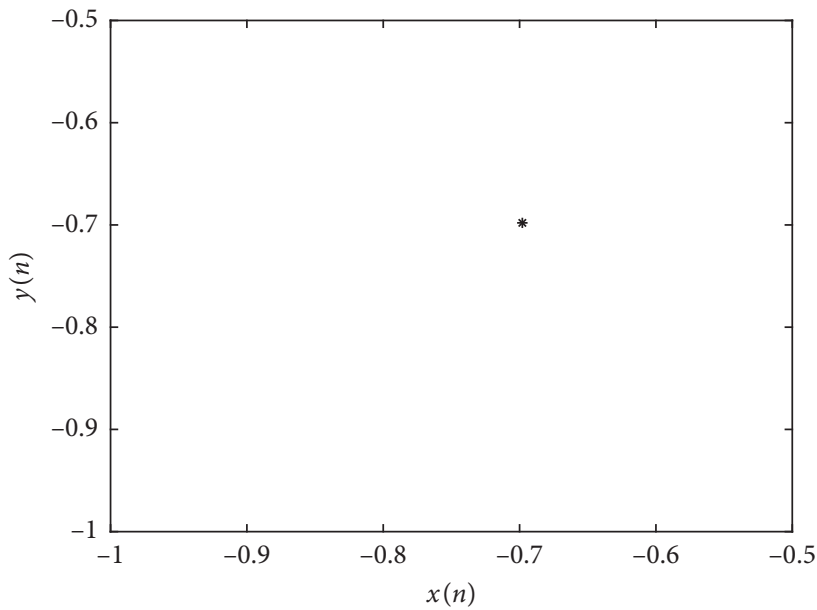

(a)

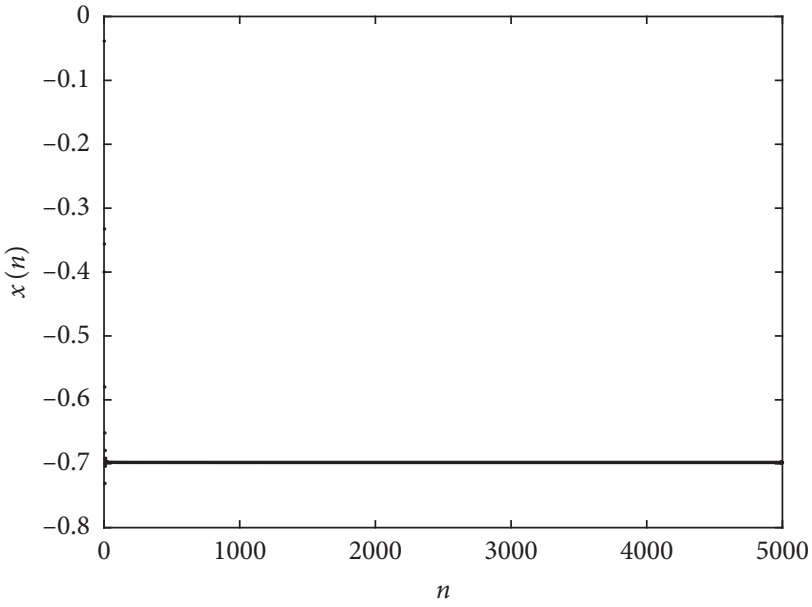

(b)

Figure 2: Continued. 


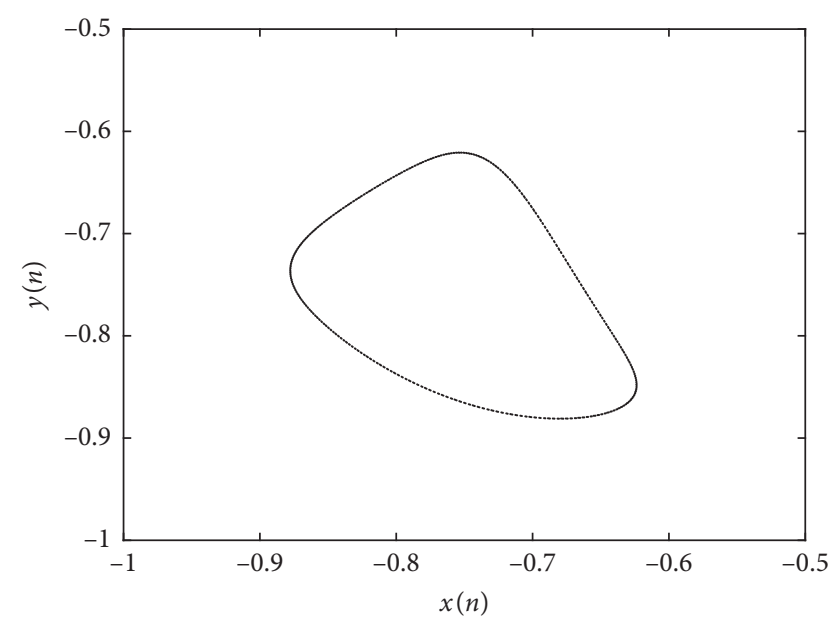

(c)

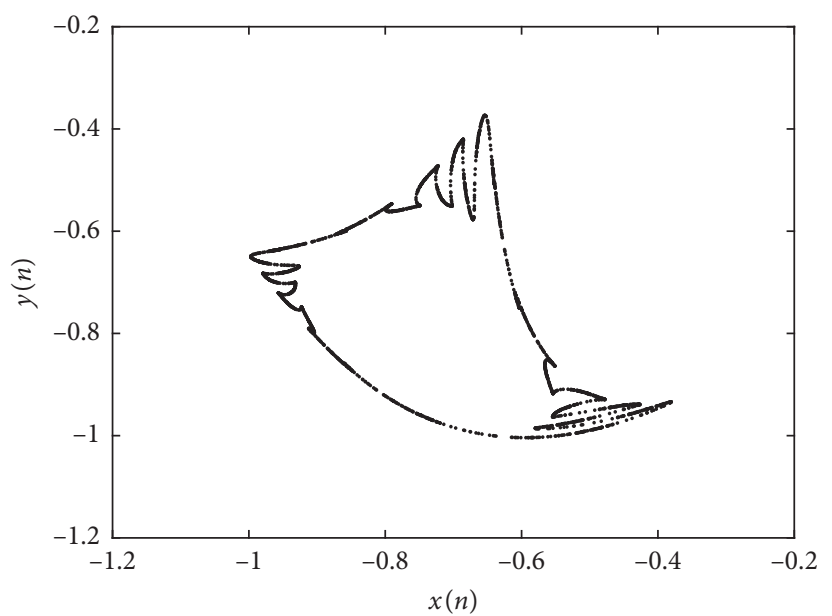

(e)

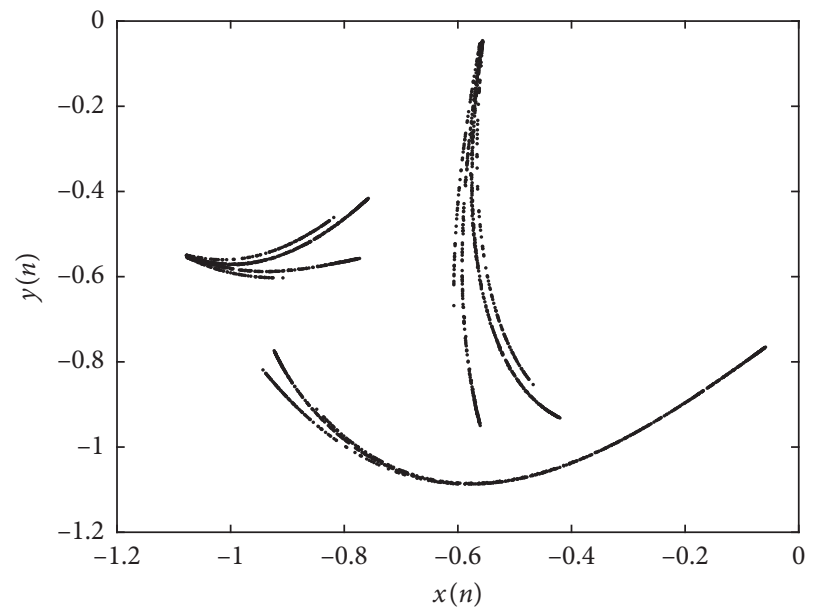

(g)

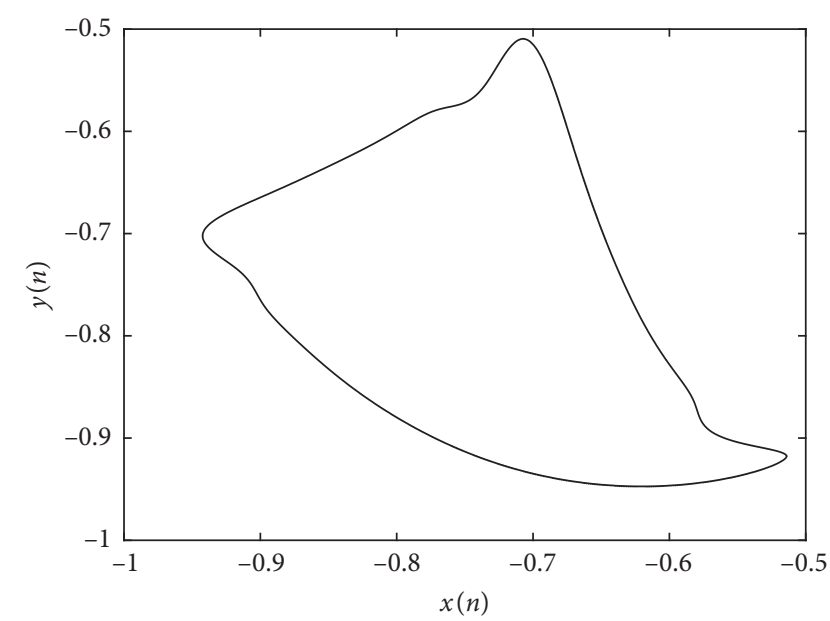

(d)

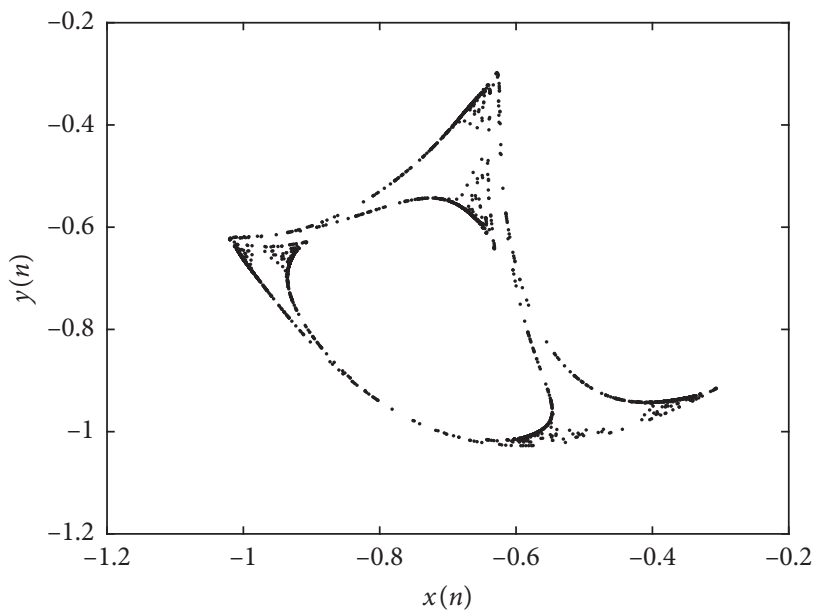

(f)

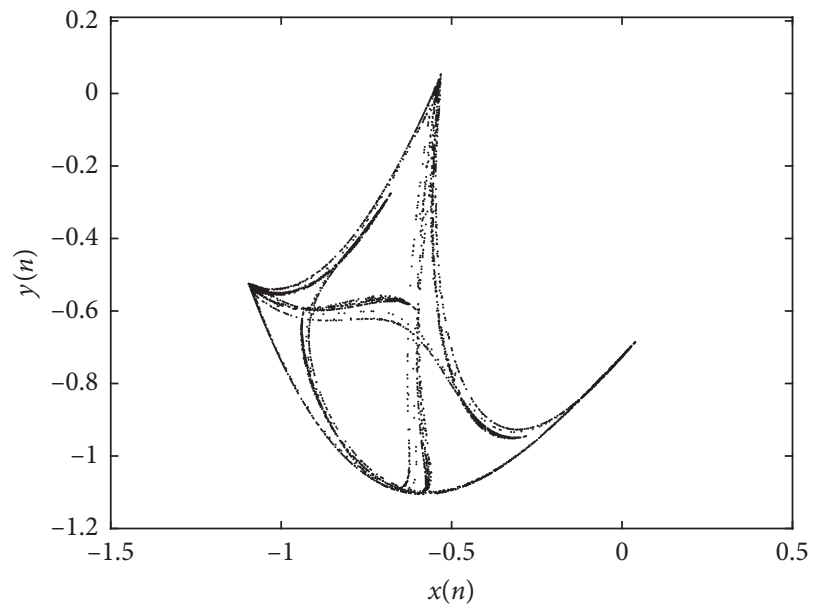

(h)

Figure 2: Continued. 


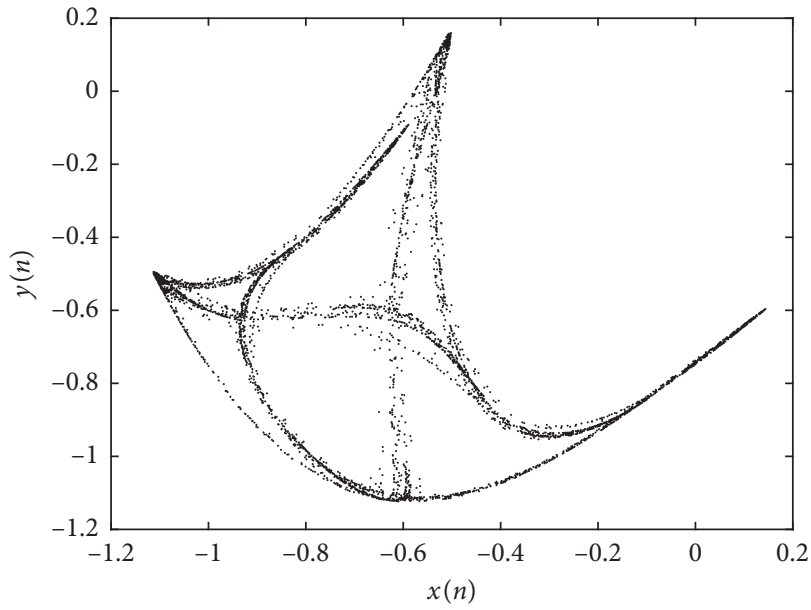

(i)

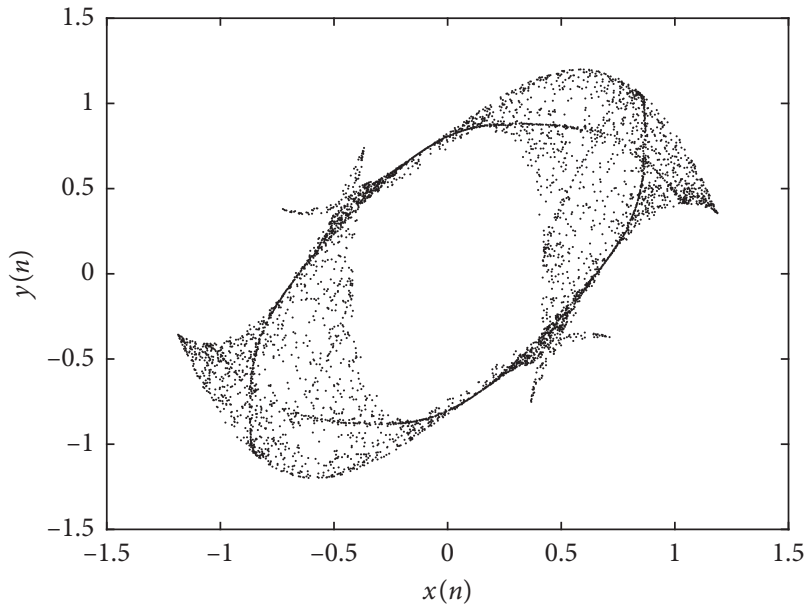

(j)

Figure 2: The phase diagrams of map (10) with the initial condition $x_{01}$ : (a) $b=1$, (b) the discrete time evolution of the state variable $x(n)$ with $b=1$, (c) $b=1.5$, (d) $b=1.6$, (e) $b=1.7$, (f) $b=1.75$, (g) $b=1.9$, (h) $b=1.95$, (i) $b=2$, and (j) $b=2.2$.

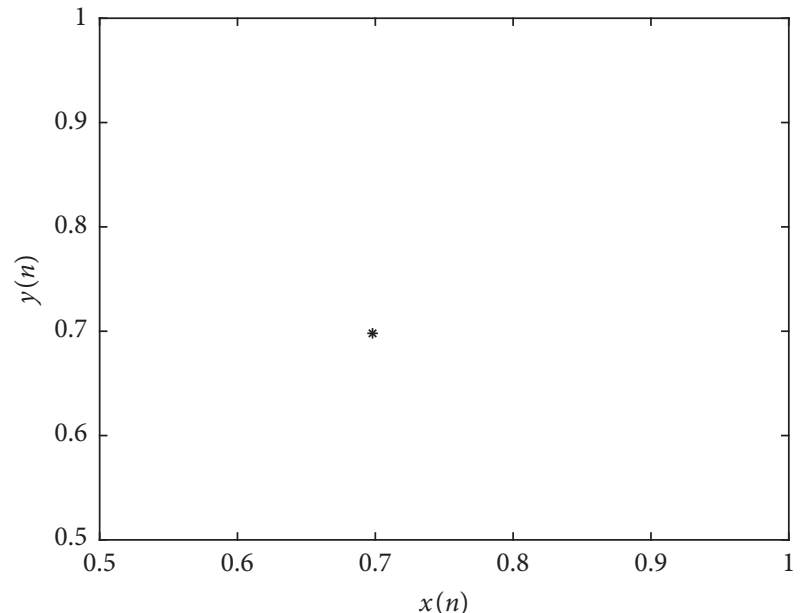

(a)

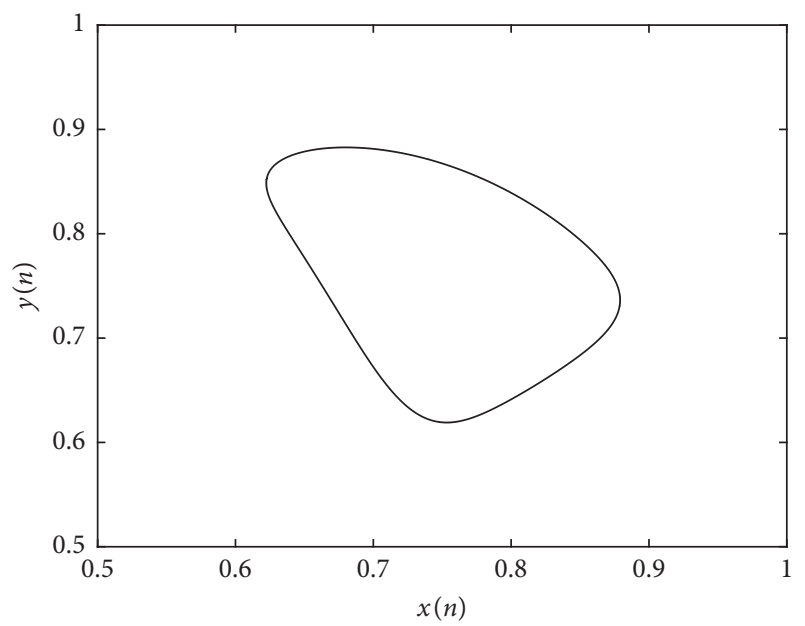

(c)

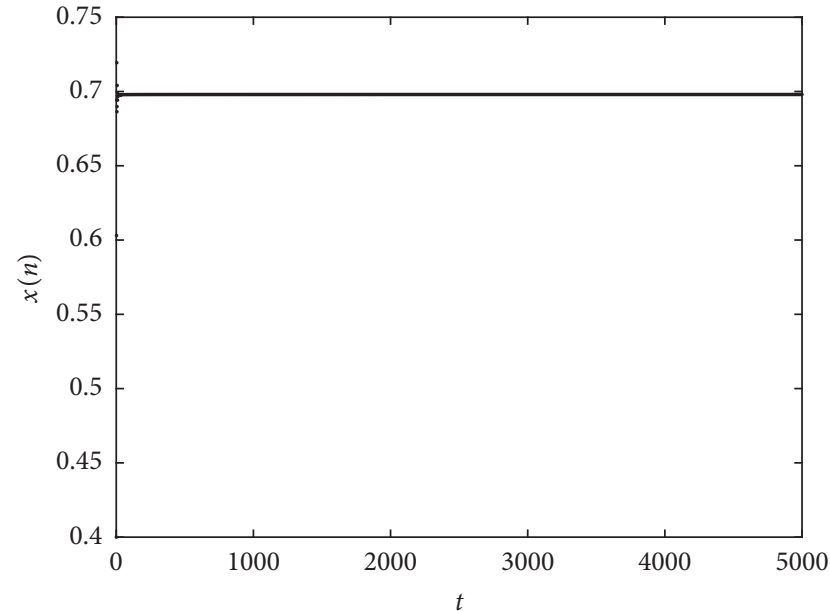

(b)

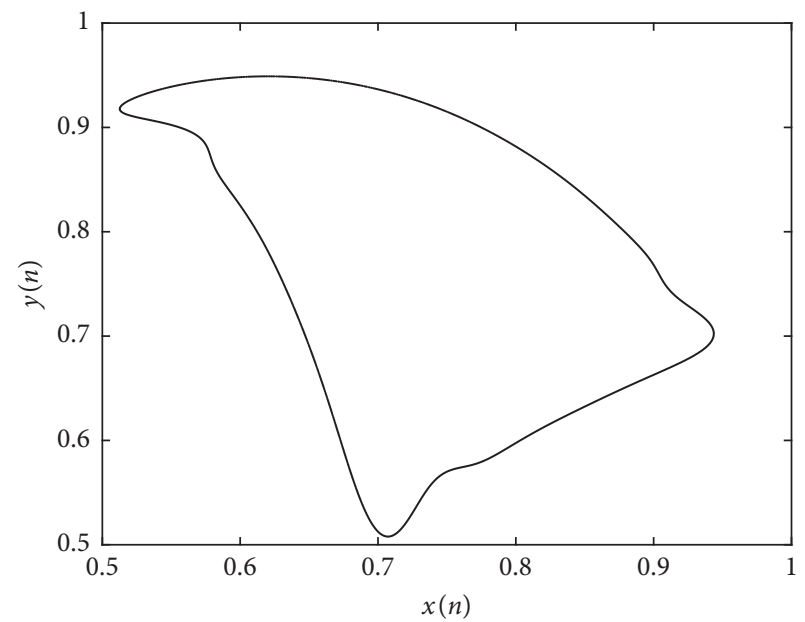

(d)

FIgURE 3: Continued. 


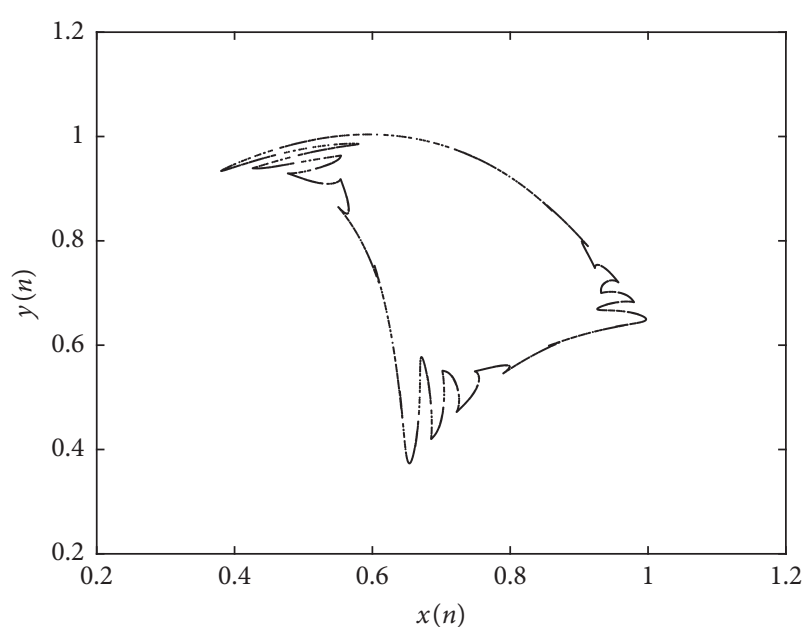

(e)

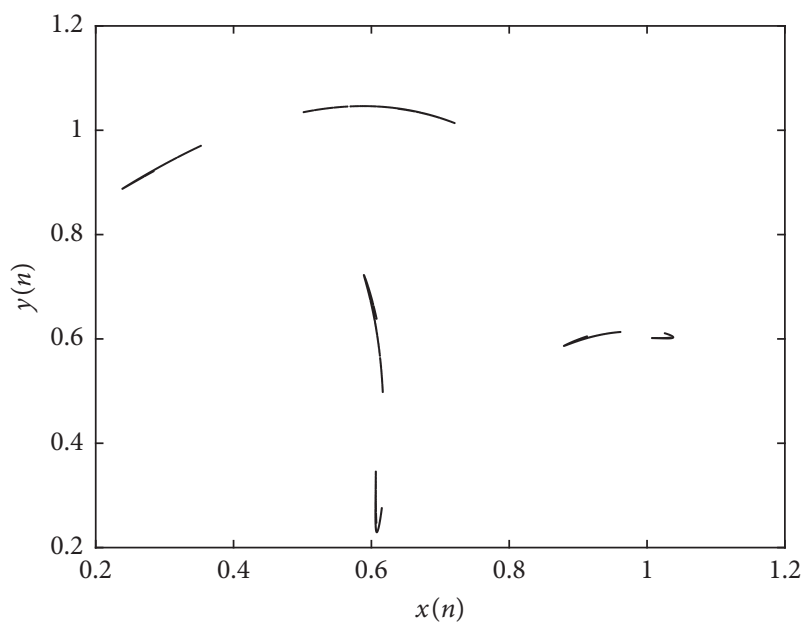

(g)

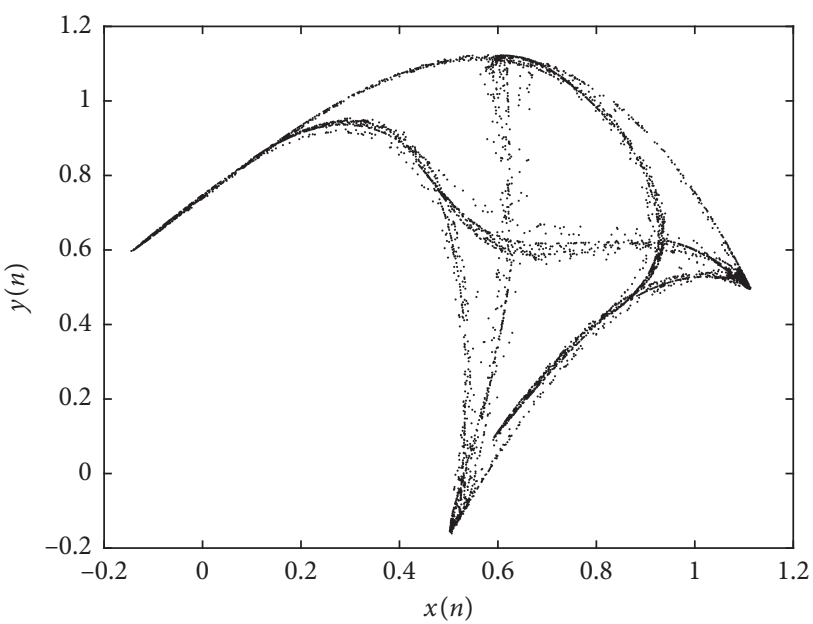

(i)

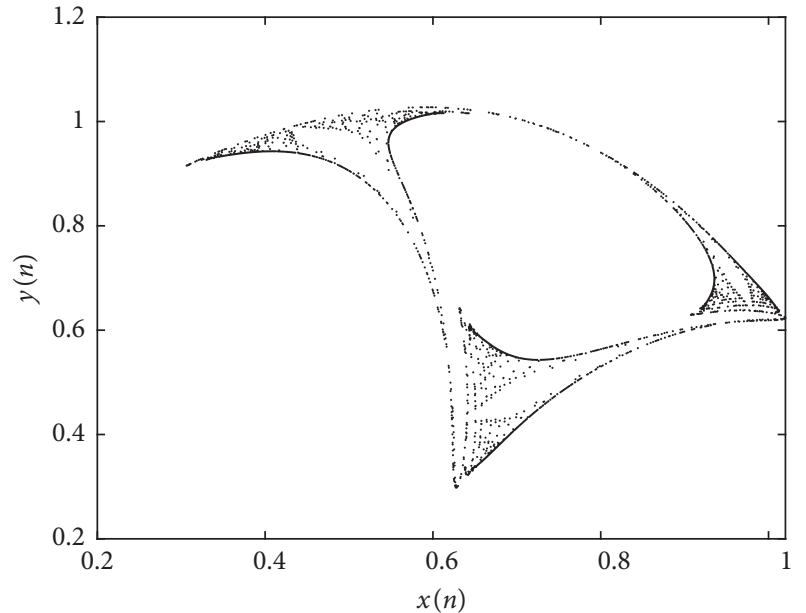

(f)

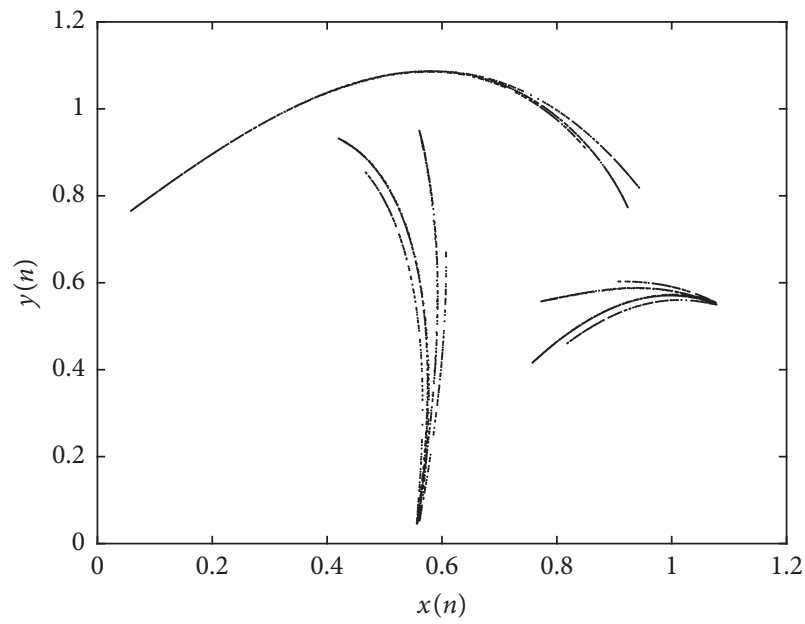

(h)

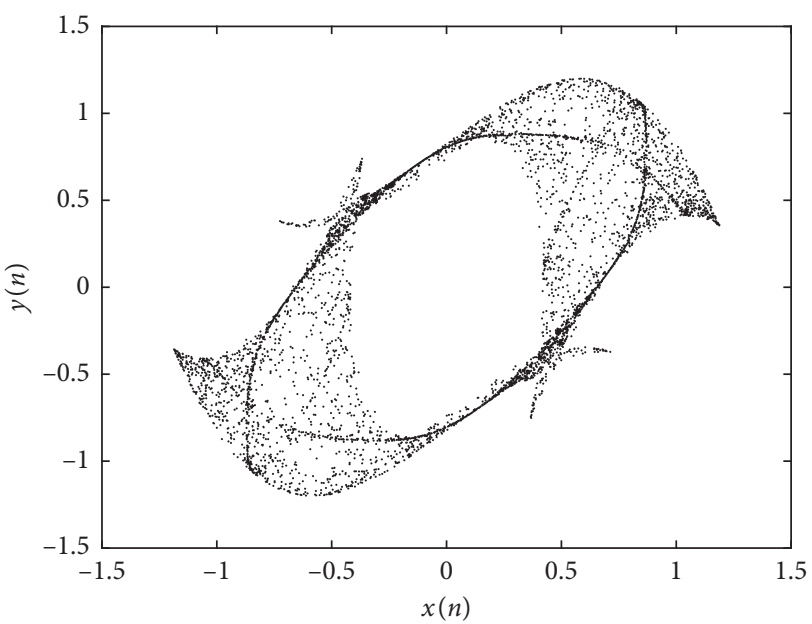

(j)

Figure 3: The phase diagrams of map (10) with the initial condition $x_{02}$ : (a) $b=1$, (b) the discrete time evolution of the state variable $x$ (n) with $b=1$, (c) $b=1.5$, (d) $b=1.6$, (e) $b=1.7$, (f) $b=1.75$, (g) $b=1.8$, (h) $b=1.9$, (i) $b=2$, and (j) $b=2.2$.

which we can see that the dynamics of the map is different from that of the map as the parameter $b$ varies and lost the symmetry.
The phase diagrams for the map with $x_{01}$ are shown in Figure 5. The map is period-2 when $c=0.2$ (Figures 5(a) and $5(\mathrm{~b})$ ), and the periodic behavior persists for a long time until 


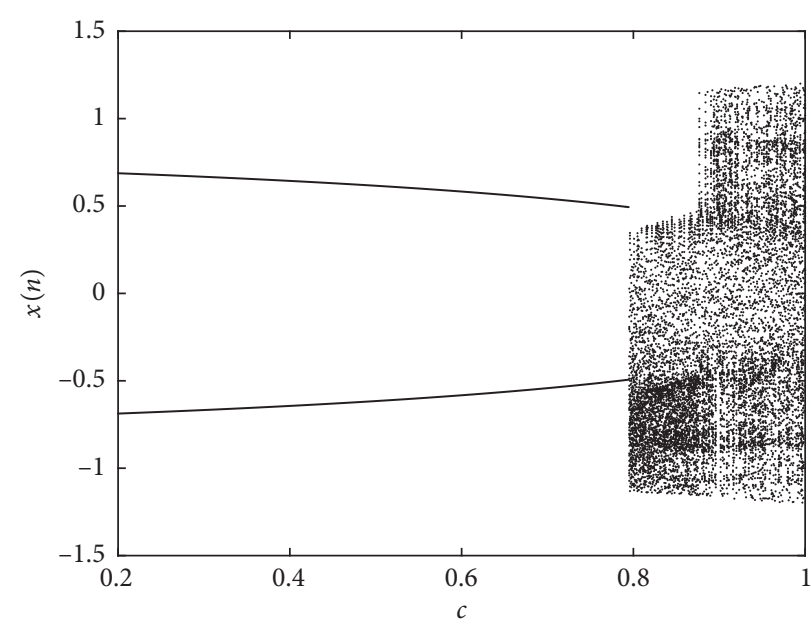

(a)

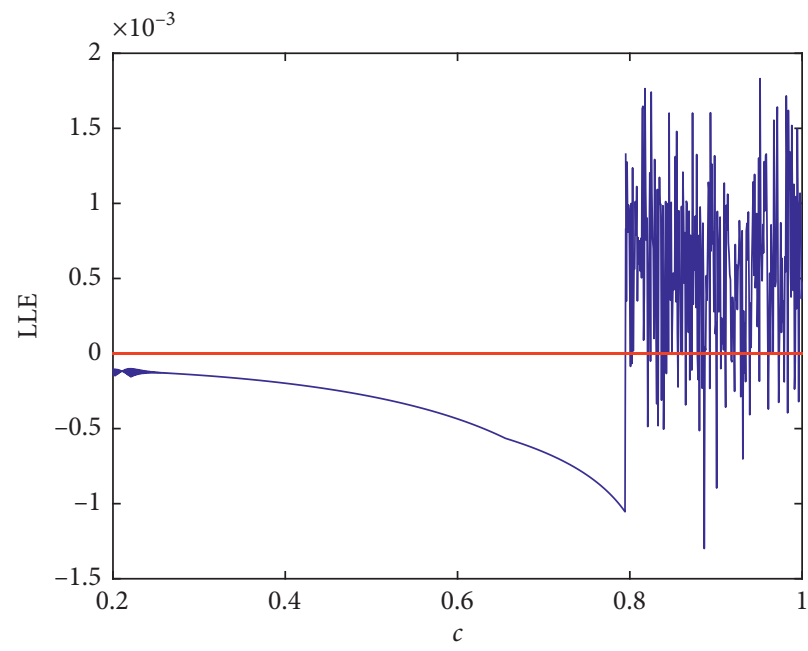

(c)

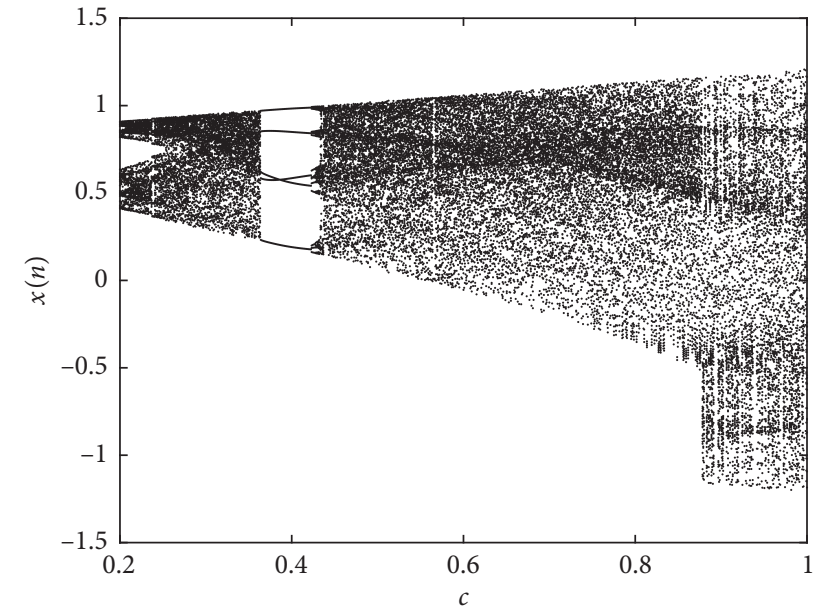

(b)

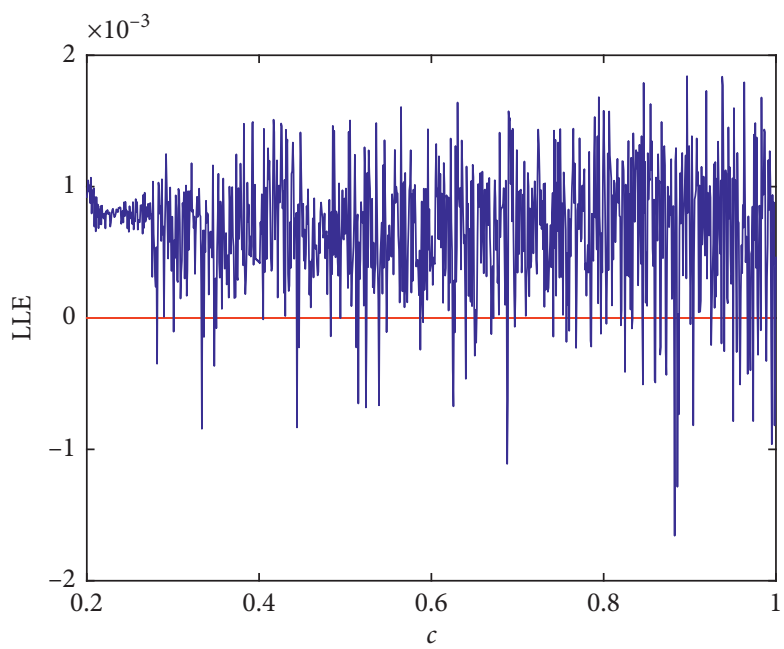

(d)

FiguRE 4: Bifurcation diagrams and corresponding LLE spectrums for the map with different initial values as the parameter $c$ varies: (a) the bifurcation diagram with $x_{01}$, (b) the bifurcation diagram with $x_{02}$, (c) the LLE spectrum with $x_{01}$, and (d) the LLE spectrum with $x_{02}$.

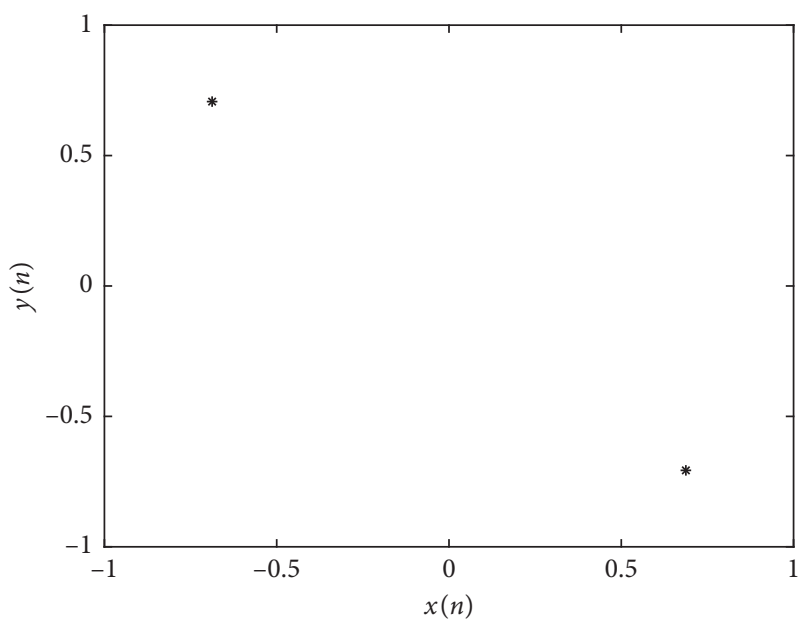

(a)

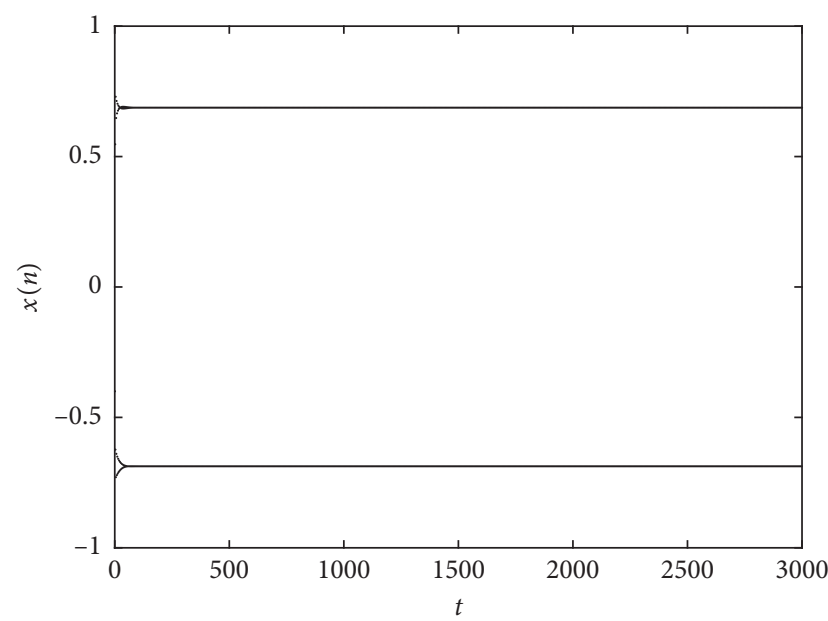

(b)

Figure 5: Continued. 


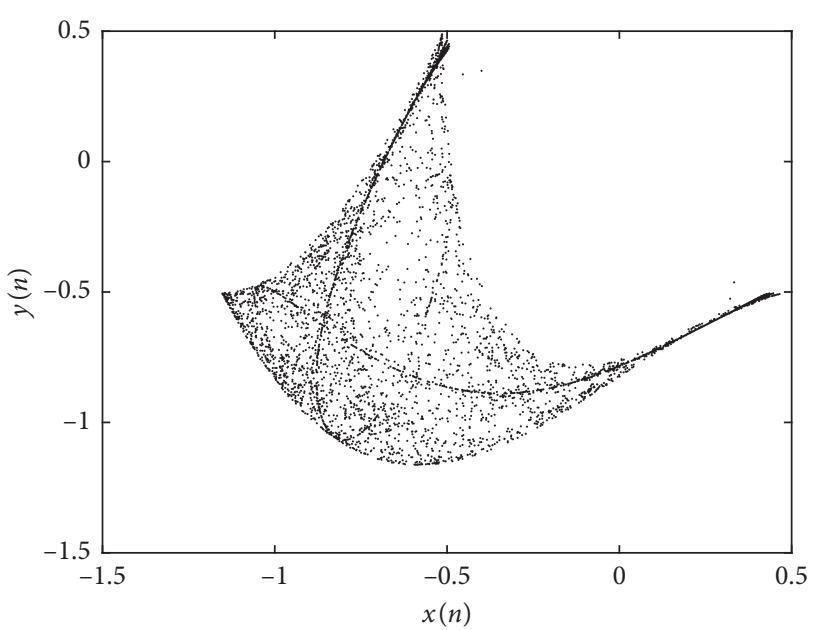

(c)

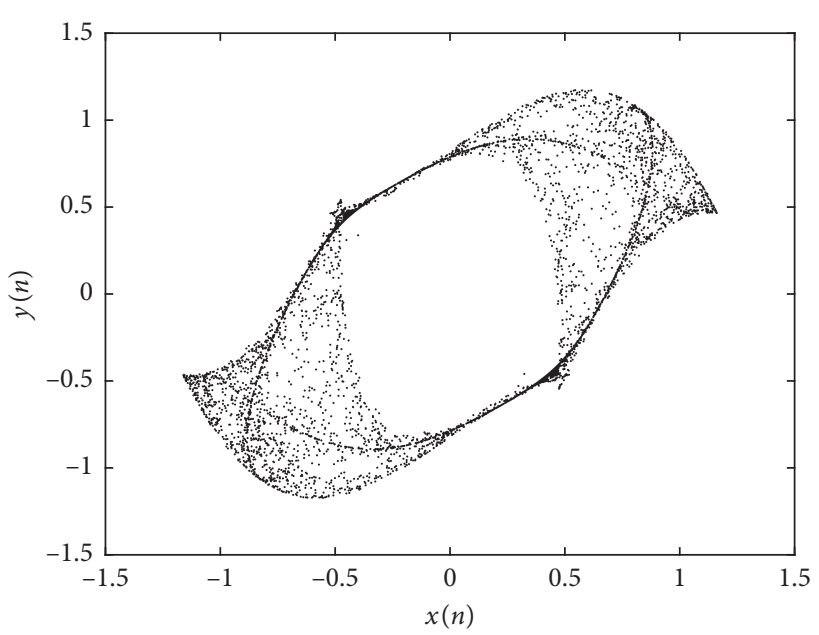

(d)

Figure 5: The phase diagrams of system (10) with the initial condition $x_{01}$ : (a) $c=0.20$, (b) the discrete time evolution of the state variable $x(n)$ with $c=0.20$, (c) $c=0.80$, and (d) $c=0.88$.

$c=0.79$. When $c=0.8$, the system is chaotic and the chaotic attractor is depicted in Figure 5(c). As $c$ increases from 0.87 to 0.88 , the chaotic attractor becomes a large one, which means an interior crisis occurs.

The dynamics of the map with the initial condition $x_{02}$ is displayed in Figure 6. The map keeps chaotic as the parameter $c$ changes in the interval of $[0.2,1]$, and the chaotic attractor has different formations. A chaotic attractor consists of four small parts in the phase space when the parameter $c$ increases from 0.2 to 0.28 (Figures 6(a)-6(c)) and converts to a whole one when $c=$ 0.29 (Figure $6(\mathrm{~d})$ ). The system is period-5 when $c=0.4$, and the phase diagram and the discrete time evolution of the state variable $x(n)$ are plotted in Figures 6(e) and 6(f). When the parameter $c$ increases from 0.87 to 0.88 , the chaotic attractor becomes a large one suddenly (Figures 6(g) and 6(h)), which implies that an interior crisis occurs.

The stable region for the map in the $b-c$ parameter plane with $v=0.98$ is plotted in Figure 7 in order to give a guidance of choosing values of the parameters. From this figure, we can see that map (10) is chaotic when $b=2.2$ and $c=0.95$.

4.3. Dynamics as the Order $v$ Varies. The parameters are fixed as $b=2.2$ and $c=0.95$, the dynamics of map (10) is studied when the order $v$ is varied in this section. The bifurcation diagrams and the corresponding LLE spectrums with $x_{01}$ and $x_{02}$ are plotted in Figure 8. From which it can be seen that the dynamics of the map also shows a symmetry with different initial conditions in this case.

For different values of the $v$, the phase diagrams with $x_{01}$ are shown in Figure 9. The map has period-1 attractor for $v=0.7$ (Figure 9(a)), and a limit cycle for $v=0.71$ (Figure 9(b)), which means a Hopf bifurcation, occurs as the order $v$ increases. The shape of the limit cycle changes as $v$ increases (Figure 9(c)). The map exists as a multicycle attractor for $v=0.83$, see Figure 9(d). As the order increases to 0.84 , the attractor becomes a chaotic one which is consisted by several small parts (Figure 9(e)). The small parts combine into one attractor when $v=0.86$ (Figure 9(f)). From Figures 9(g) and 9(h), it is clear that the chaotic attractor has three small parts in the phase plane when $v=0.89$, and these parts become a whole one when $v=0.9$. The chaotic attractor in Figure 9(i) becomes a large one (Figure $9(j)$ ) when the order varies from 0.95 to 0.96 .

The phase diagrams with initial condition $x_{02}$ as the order varies from 0.7 to 0.96 are shown in Figure 10, which are symmetric with those of the map with $x_{01}$. From the global dynamics perspective, the two chaotic attractors, which are depicted in Figures 9(i) and 10(i), collide with each other and convert to a large one (Figures $9(\mathrm{j})$ and $10(\mathrm{j}))$.

\subsection{Bifurcation with the Simultaneous Variation of Both} Parameter and Order $v$. In this section, the bifurcations of map (10) as a parameter and the order variation with the initial conditions $x_{01}$ and $x_{02}$ are studied. Firstly, the value of parameter $c$ is fixed as 0.95 . The bifurcation diagram of the map is depicted in Figure 11, when the parameter $b \in[0.2,2.2]$ and the order $v \in[0.5,1]$ change simultaneously. Secondly, the value of parameter $b$ is set as 2.2 . Figure 12 shows the bifurcation diagram of the map with the variation of the parameter $b \in[0.2,2.2]$ and the order $v \in[0.5,1]$. From these figures, it can be seen that map (10) is periodic when the order is less than a certain threshold and appears chaotic behavior when the order is greater than the certain threshold.

In other words, the dynamics of map (10) becomes regular as the derivate order $v$ decreases from 1 to 0.5 and complex as the derivate order $v$ increases from 0.5 to 1 . 


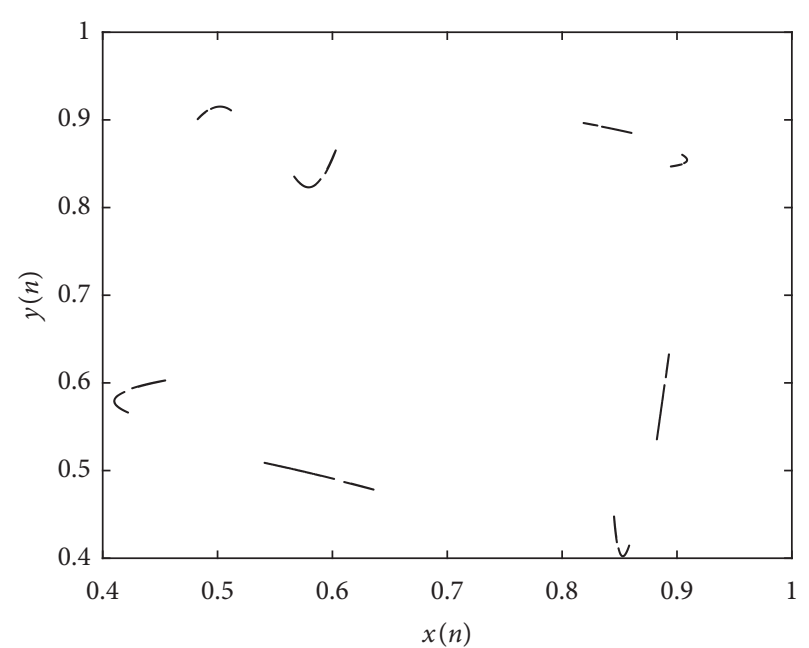

(a)

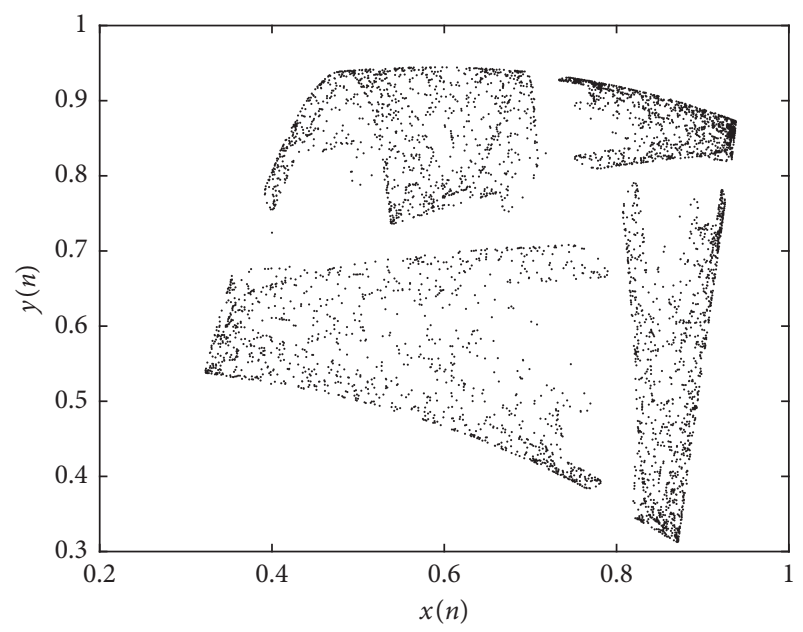

(c)

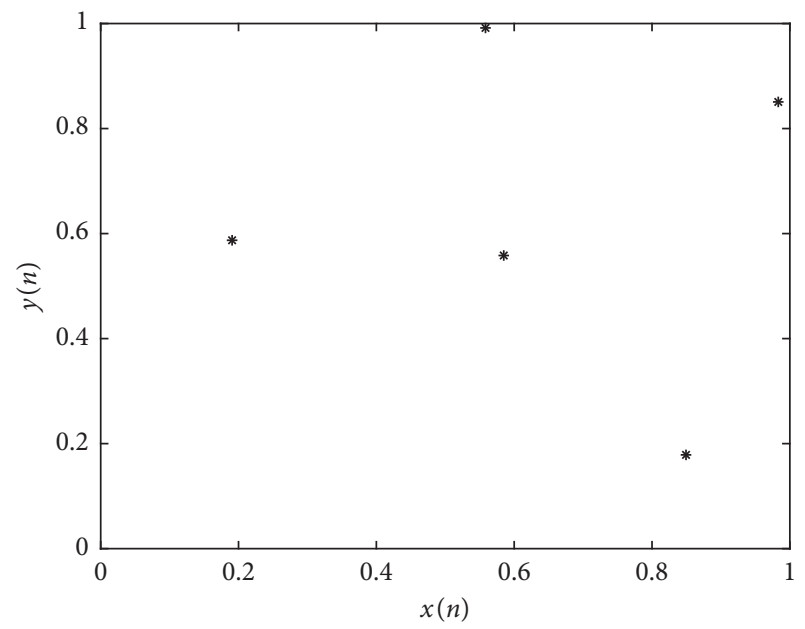

(e)

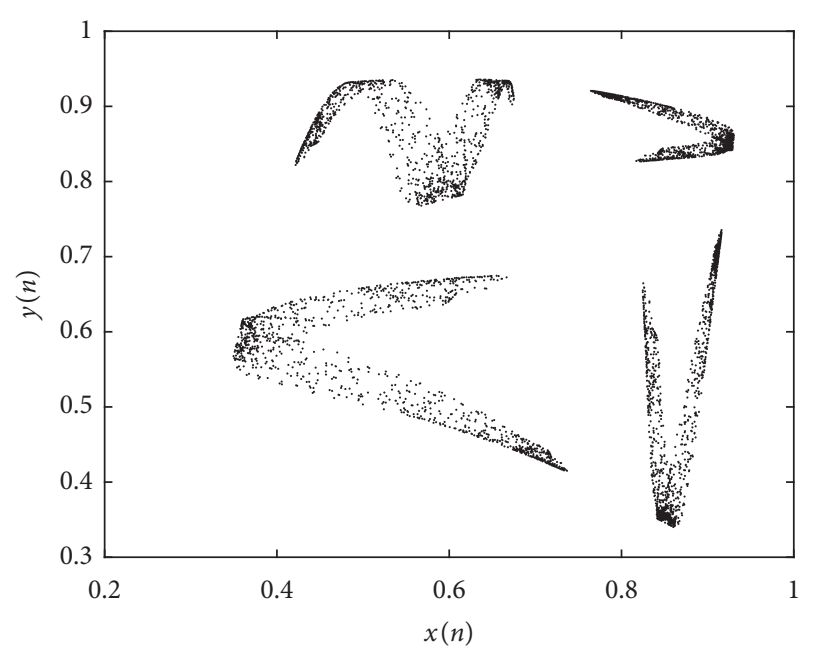

(b)

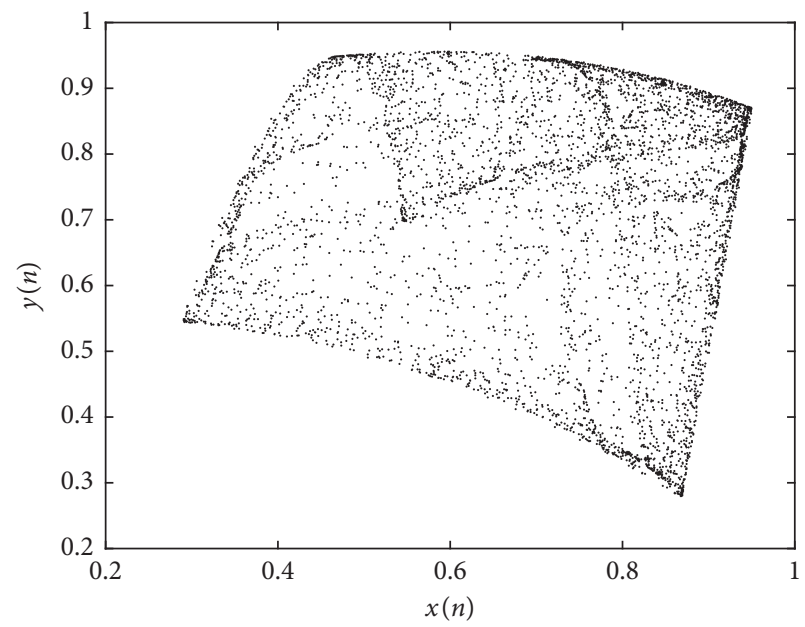

(d)

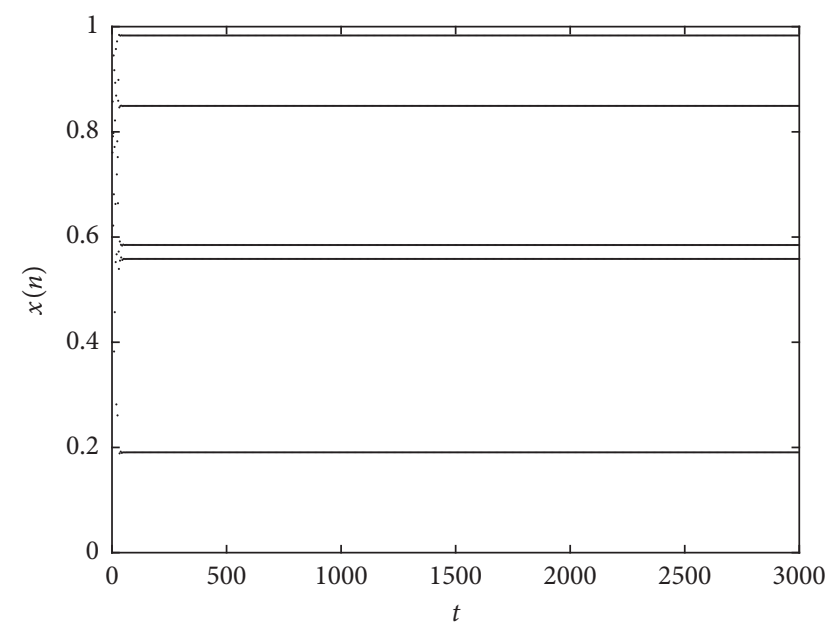

(f)

Figure 6: Continued. 


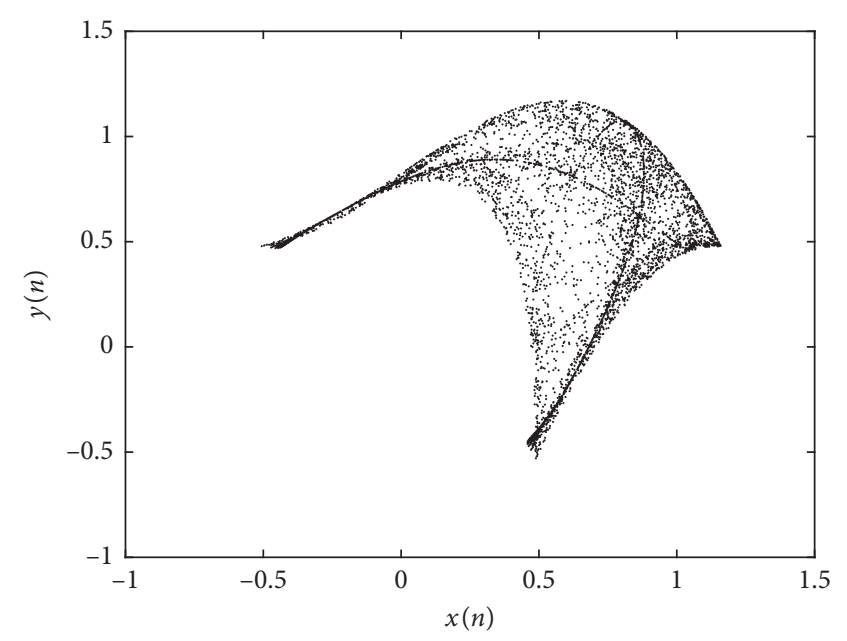

(g)

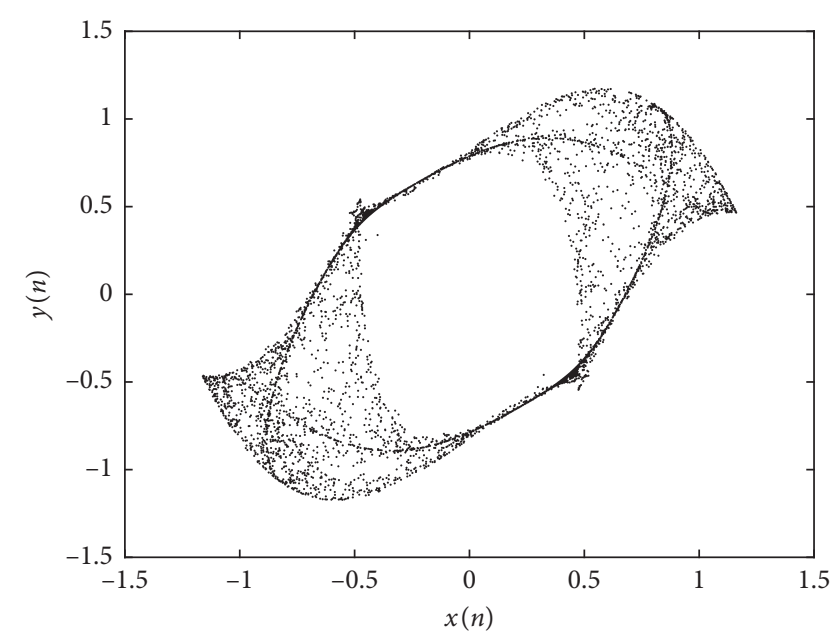

(h)

Figure 6: The phase diagrams of system (10) with the initial condition $x_{02}$ : (a) $c=0.20$ (b) $c=0.25$, (c) $c=0.28$, (d) $c=0.29$, (e) $c=0.40$, (f) discrete time evolution of the state variable $x(n)$ with $c=0.40,(\mathrm{~g}) c=0.87$, and $(\mathrm{h}) c=0.88$.

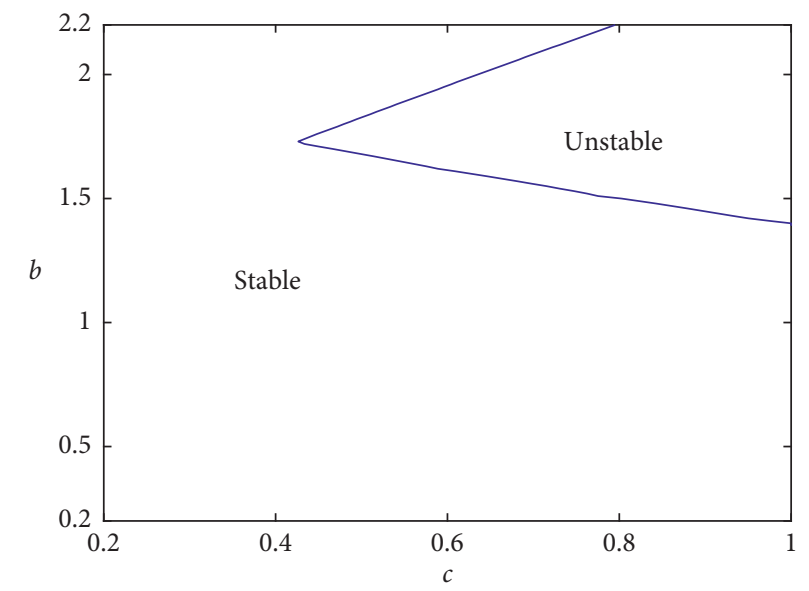

Figure 7: The stable region of the map for the two parameters.

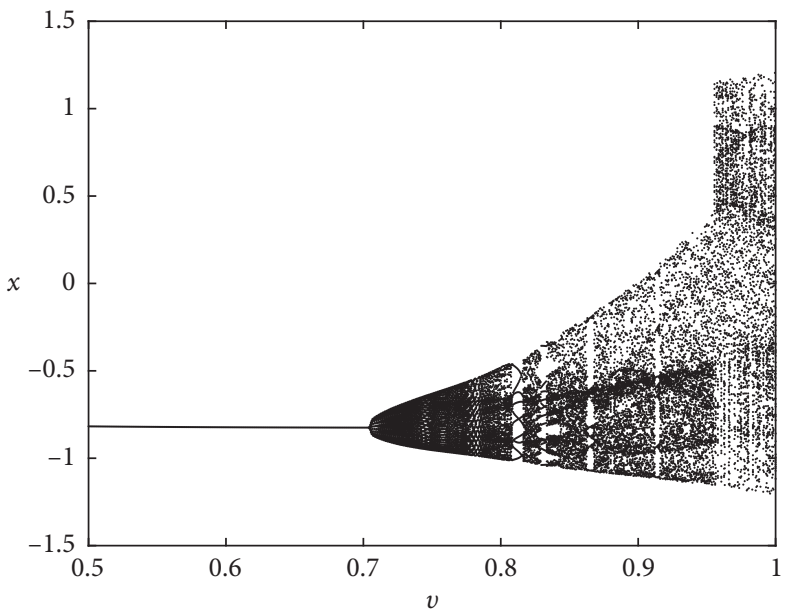

(a)

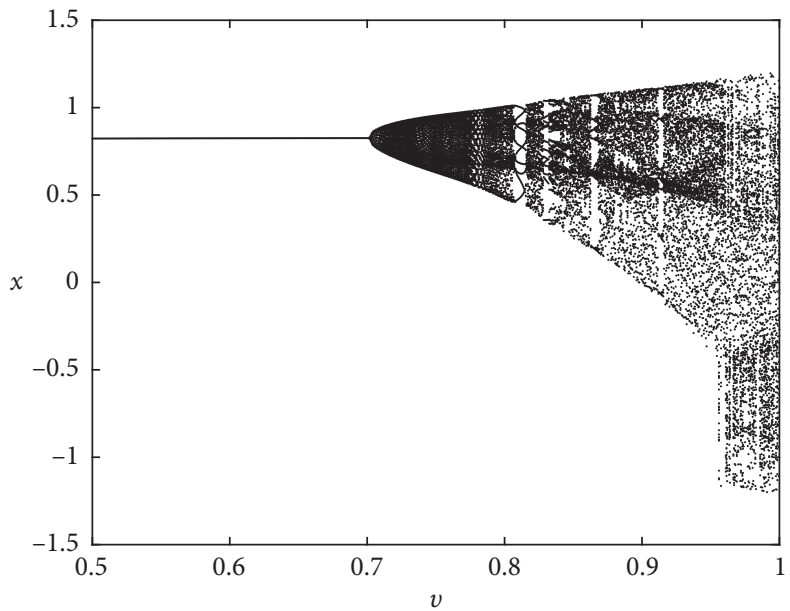

(b)

Figure 8: Continued. 


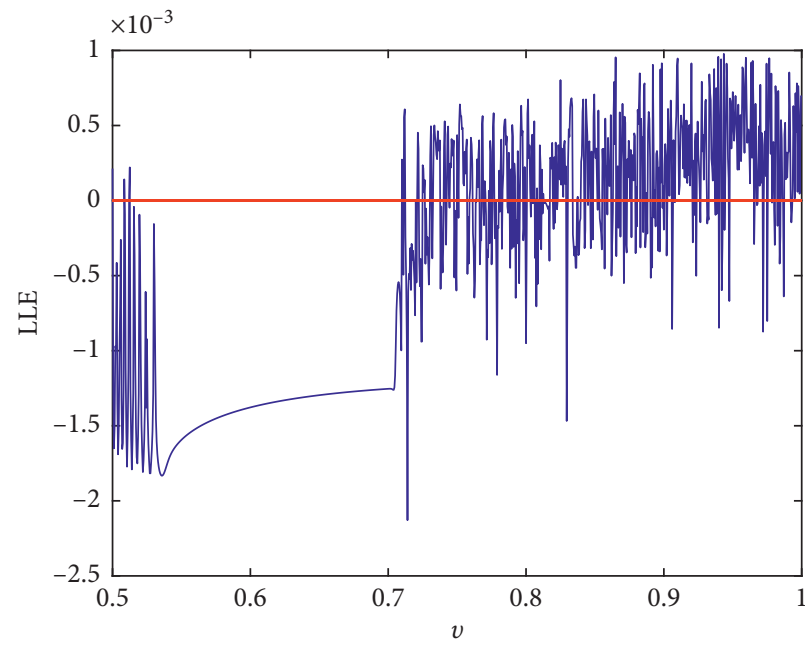

(c)

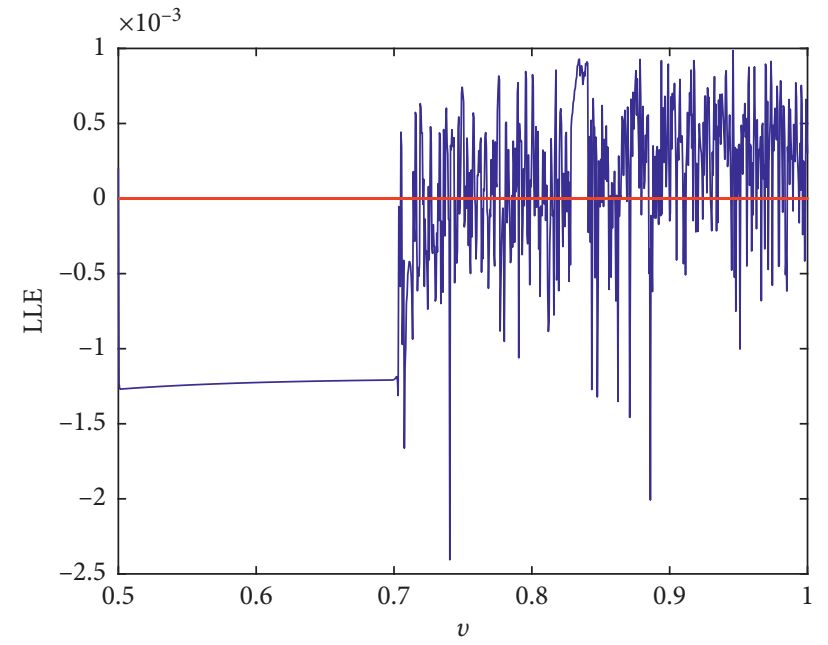

(d)

FIGURE 8: Bifurcation diagrams and corresponding LLE spectrums for the map with different initial values as the order $v$ varies: (a) the bifurcation diagram with $x_{01}$, (b) the bifurcation diagram with $x_{02}$, (c) the LLE spectrum with $x_{01}$, and (d) the LLE spectrum with $x_{02}$.

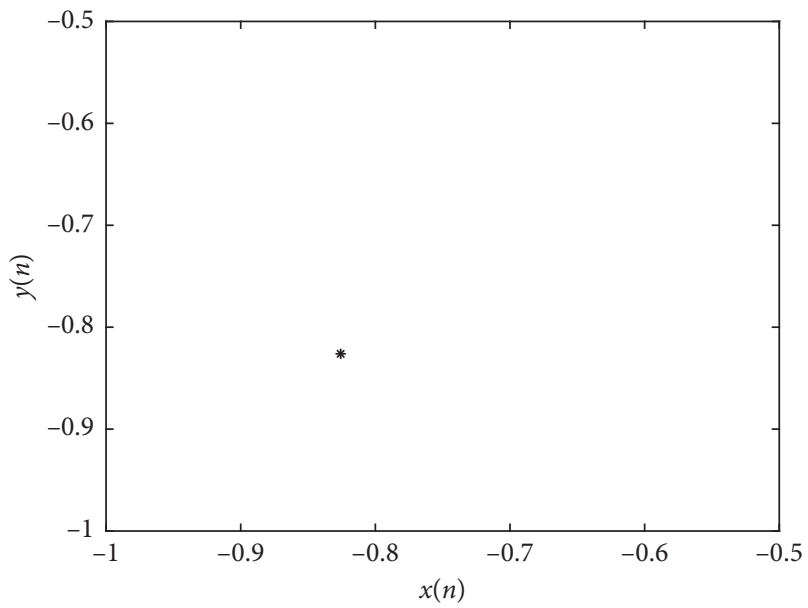

(a)

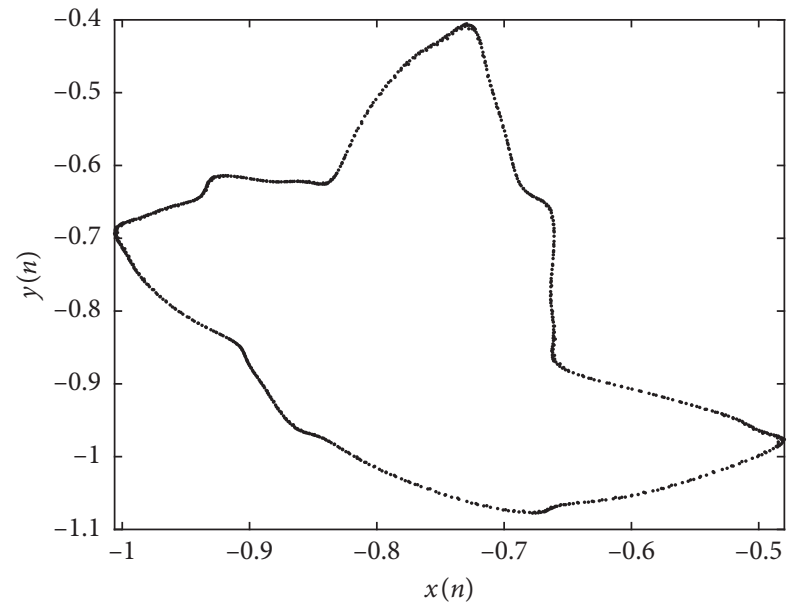

(c)

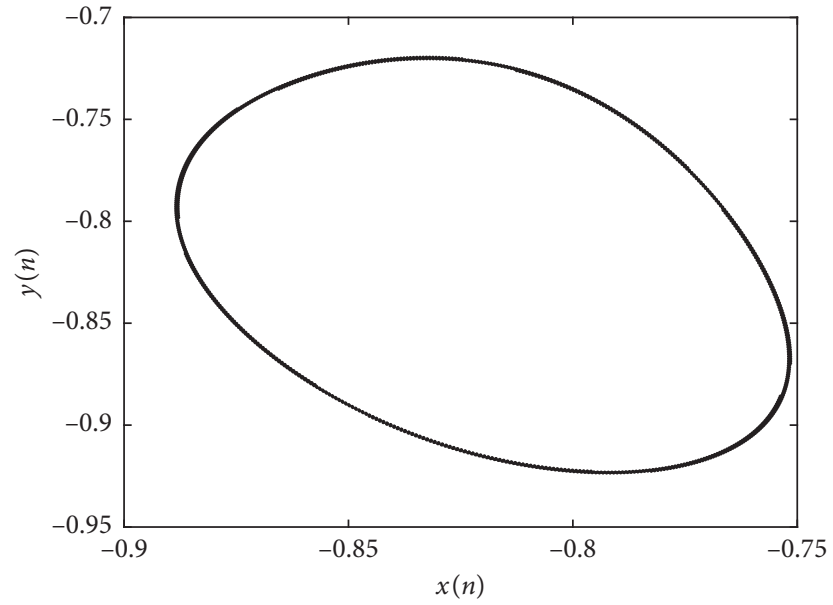

(b)

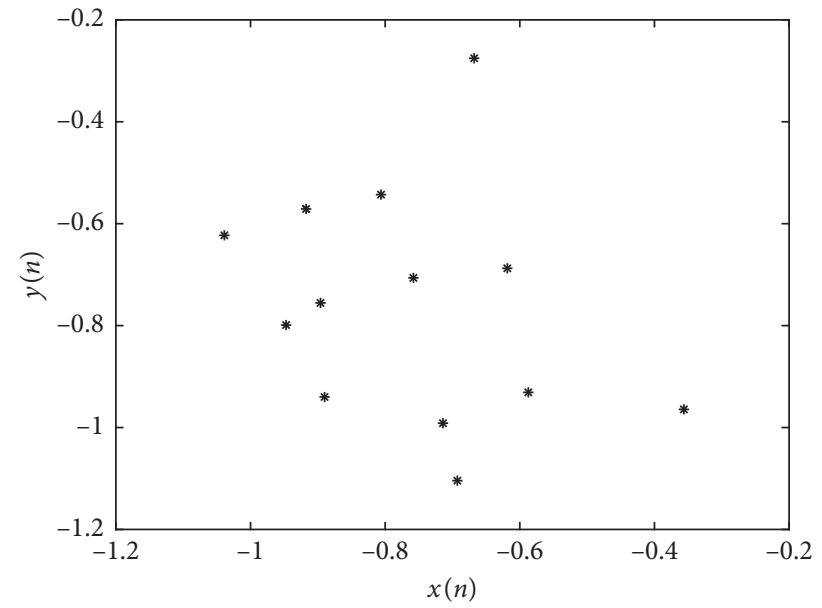

(d)

Figure 9: Continued. 


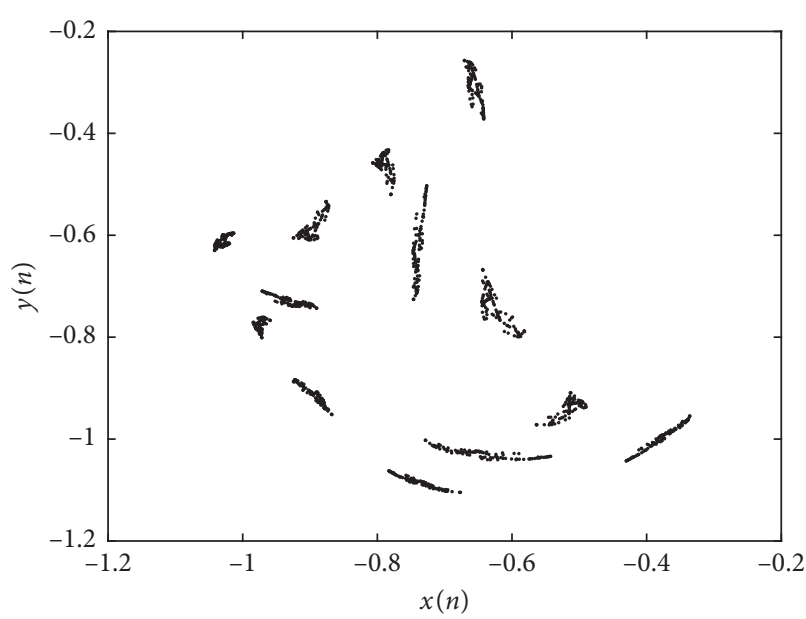

(e)

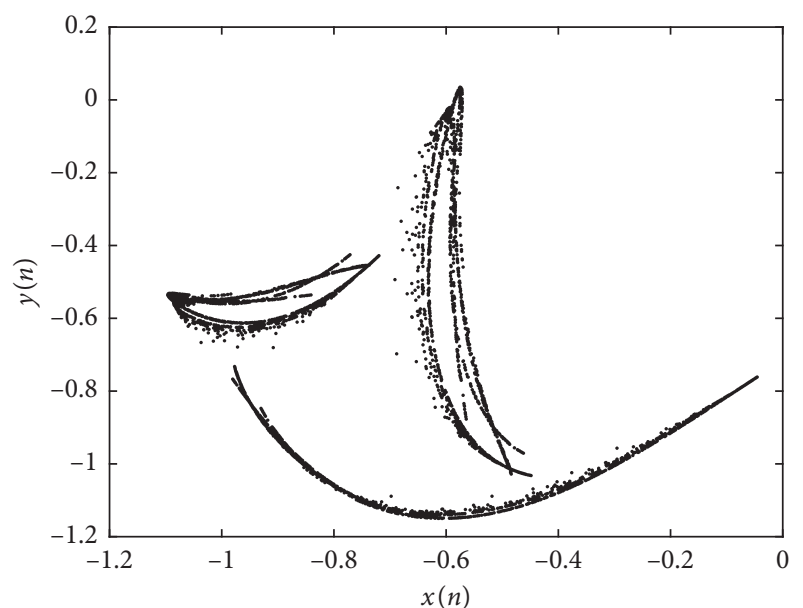

(g)

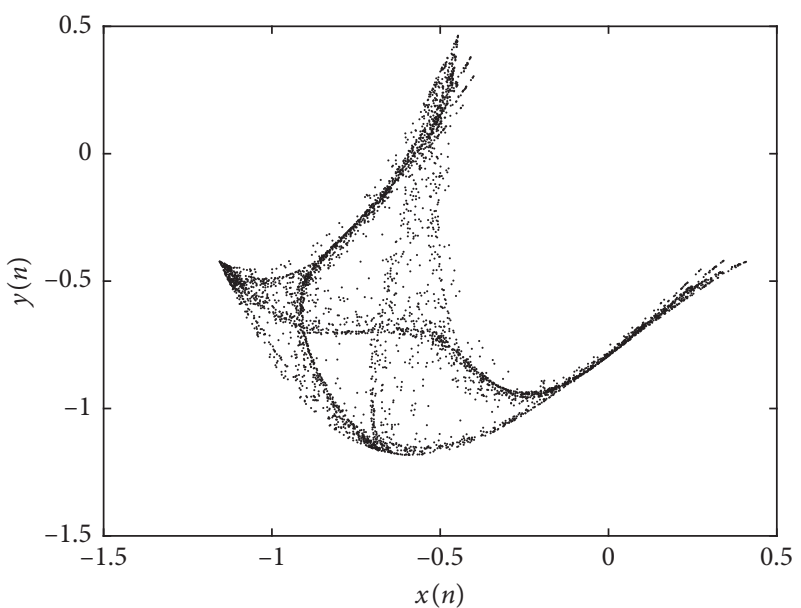

(i)

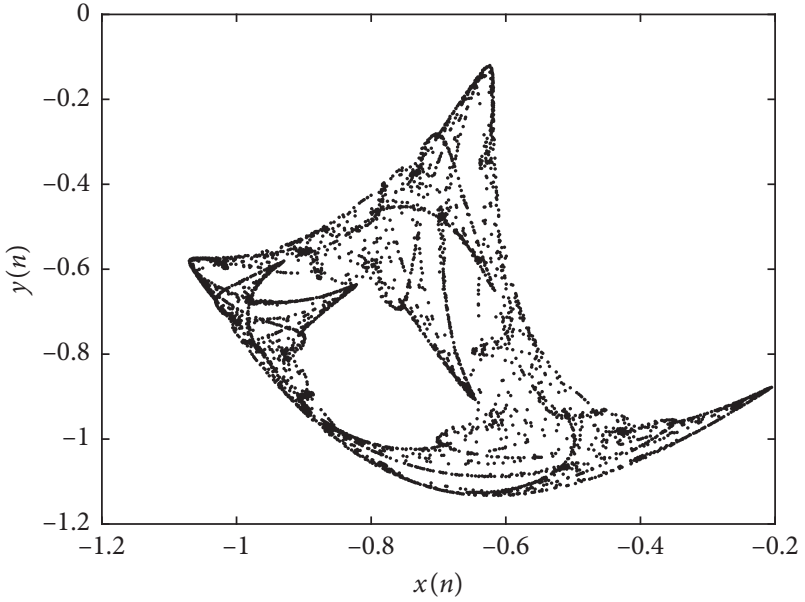

(f)

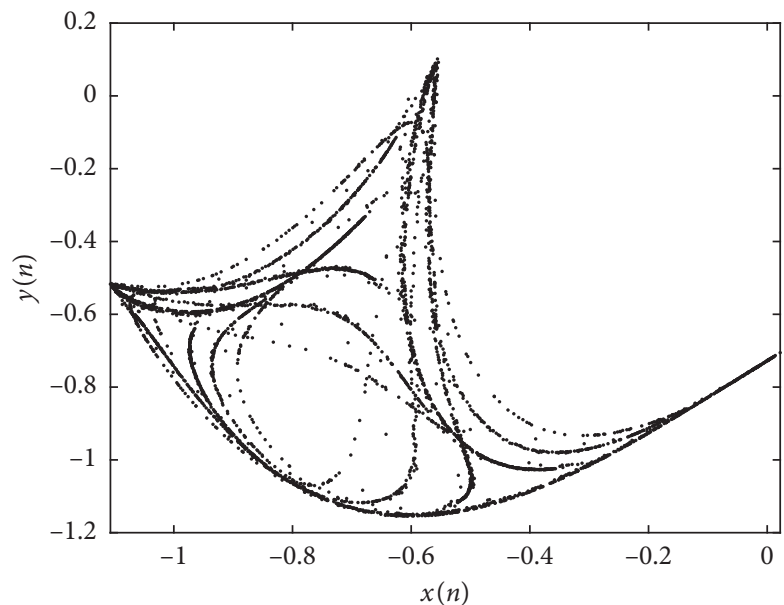

(h)

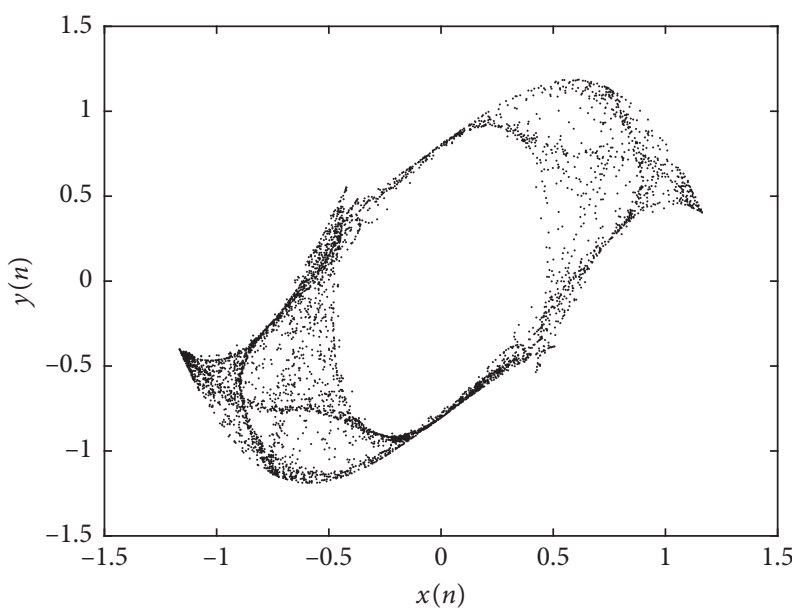

(j)

Figure 9: The phase diagrams of system (10) with the initial condition $x_{01}:$ (a) $v=0.70$, (b) $v=0.71$, (c) $v=0.81$, (d) $v=0.83$, (e) $v=0.84$, (f) $v=0.86$, (g) $v=0.89$, (h) $v=0.90$, (i) $v=0.95$, and (j) $v=0.96$. 


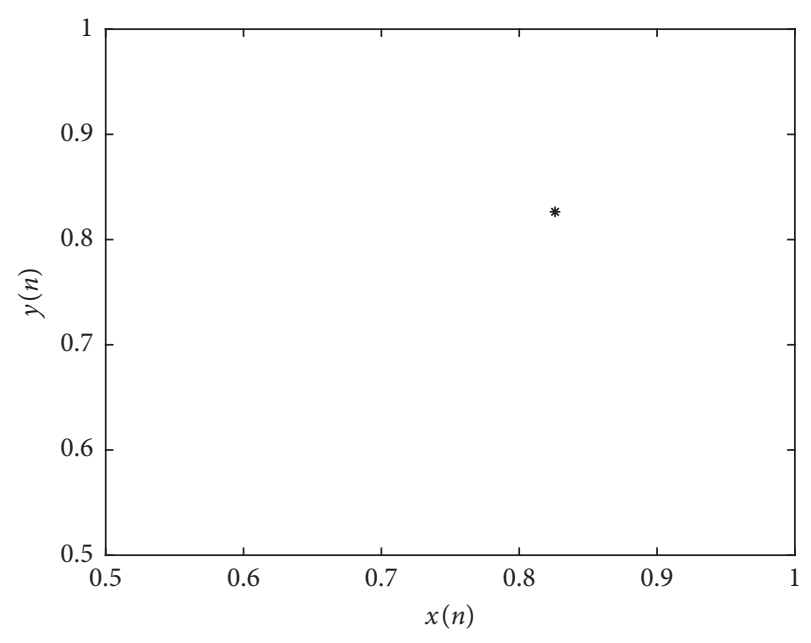

(a)

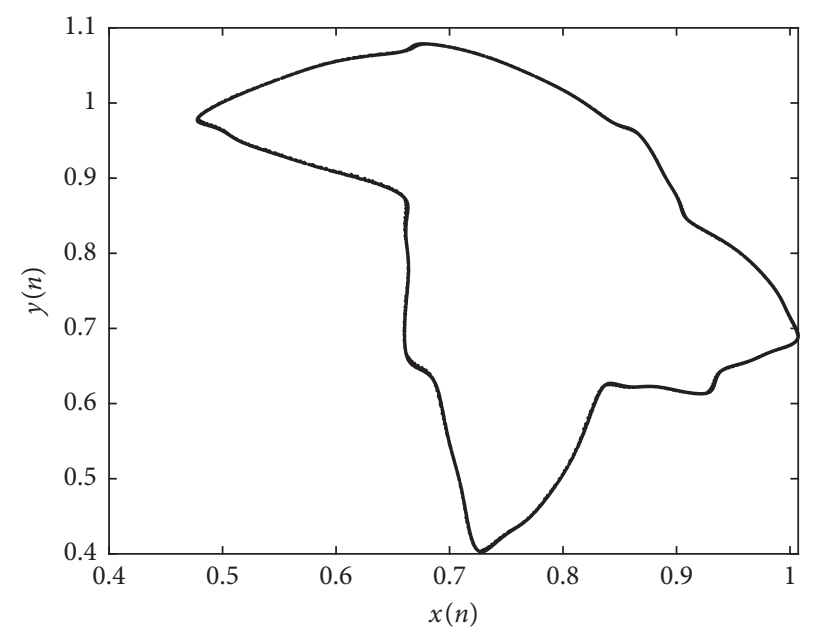

(c)

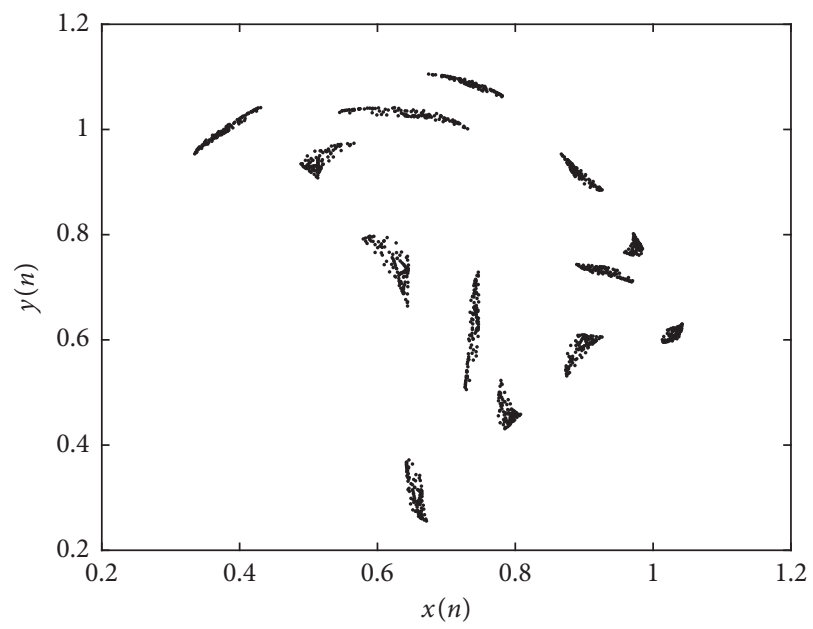

(e)

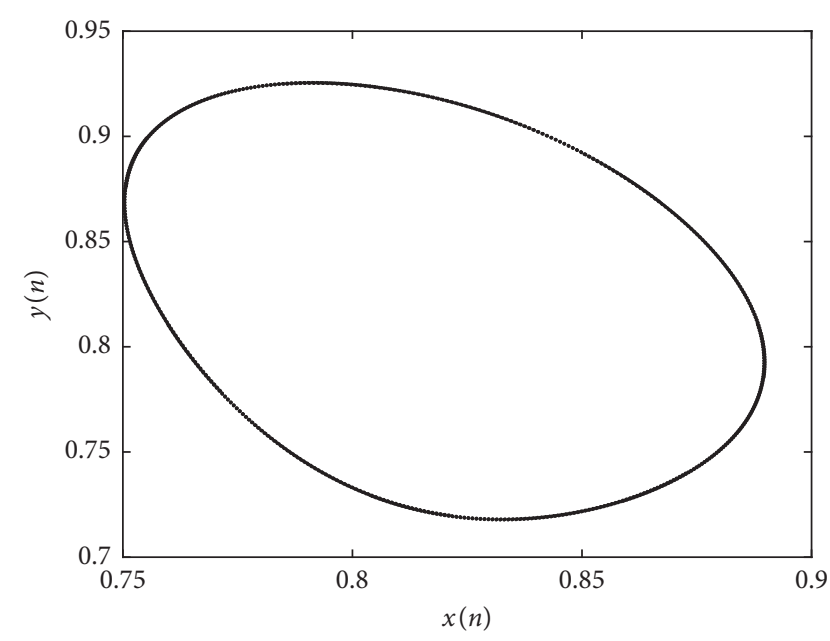

(b)

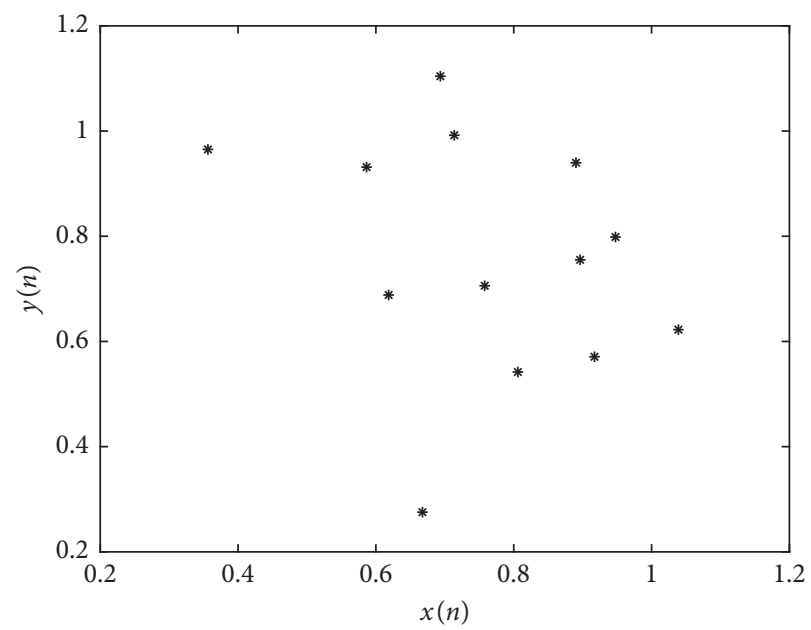

(d)

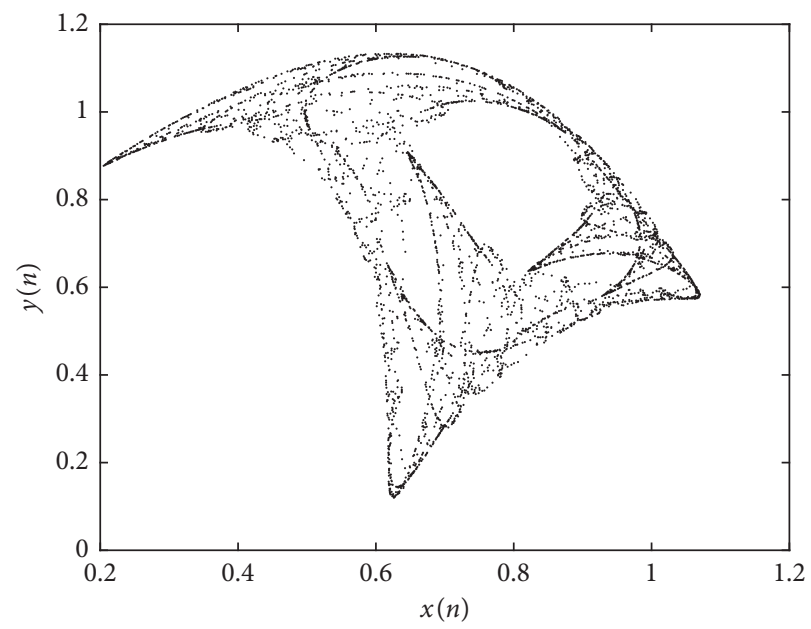

(f)

Figure 10: Continued. 


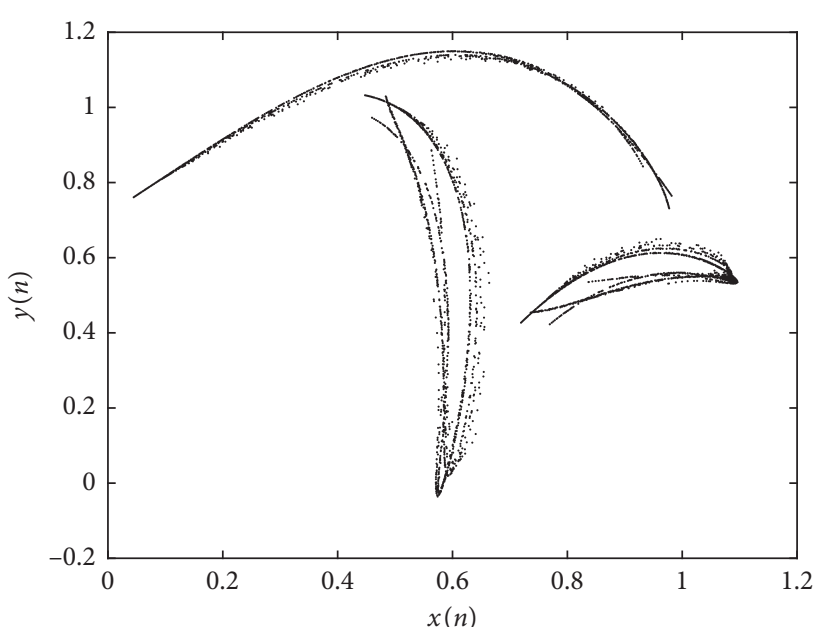

(g)

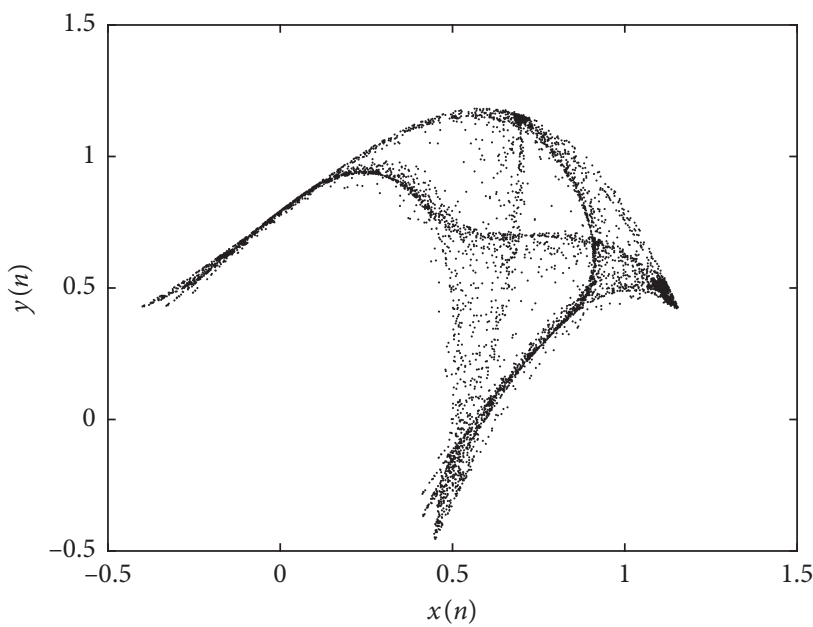

(i)

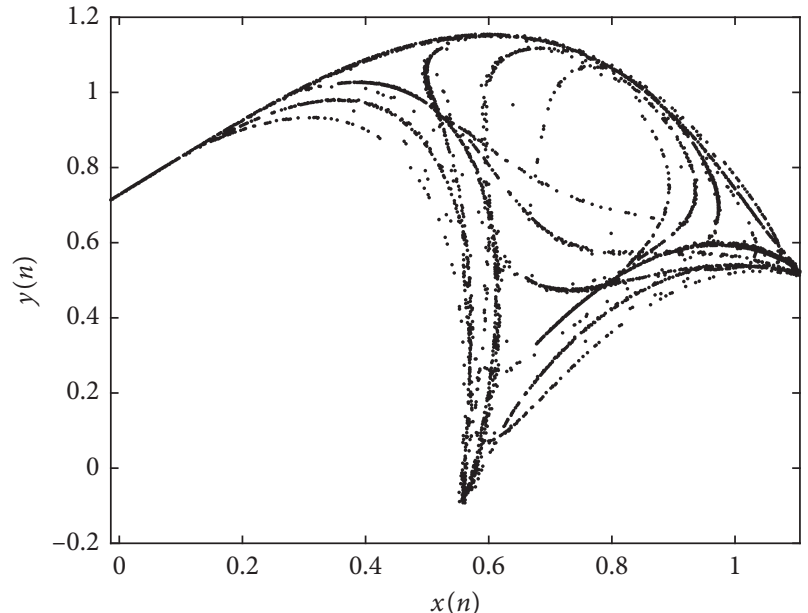

(h)

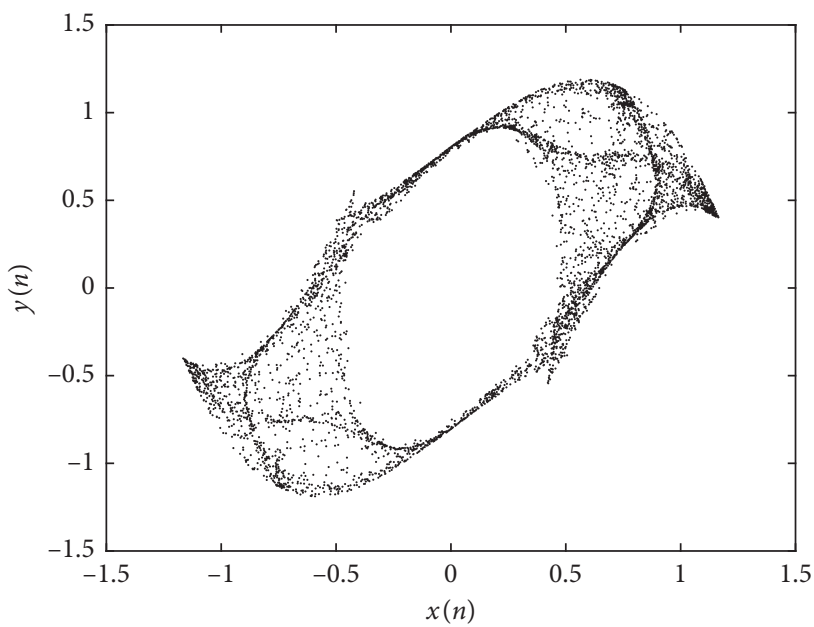

(j)

FIGURE 10: The phase diagrams of system (10) with the initial condition $x_{02}$ : (a) $v=0.70$, (b) $v=0.71$, (c) $v=0.80$, (d) $v=0.83$, (e) $v=0.84$, (f) $v=0.86$, (g) $v=0.89$, (h) $v=0.90$, (i) $v=0.95$, and (j) $v=0.96$.

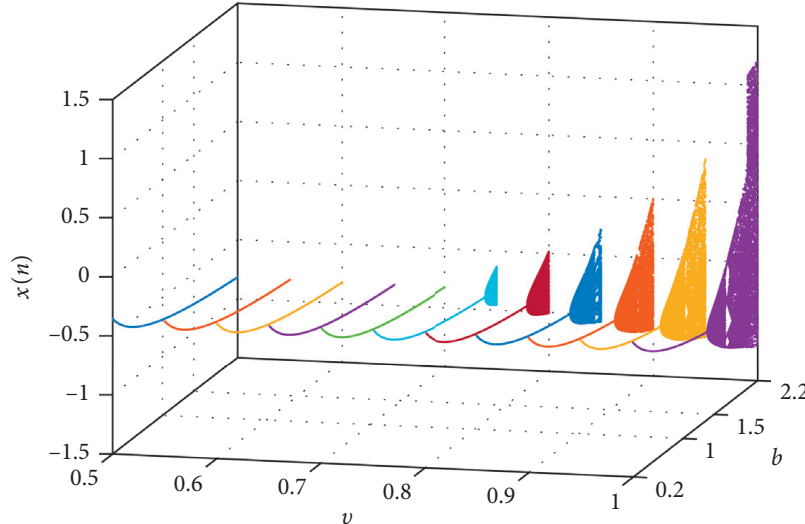

(a)

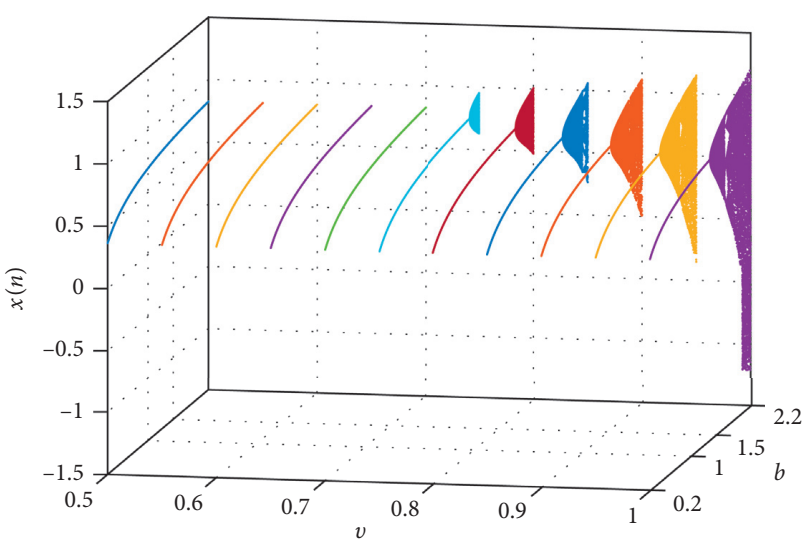

(b)

FiguRE 11: Bifurcation diagrams for map (10) with different initial values as the parameter $b$ and the order $v$ vary: (a) the bifurcation diagram with $x_{01}$ and (b) the bifurcation diagram with $x_{02}$. 


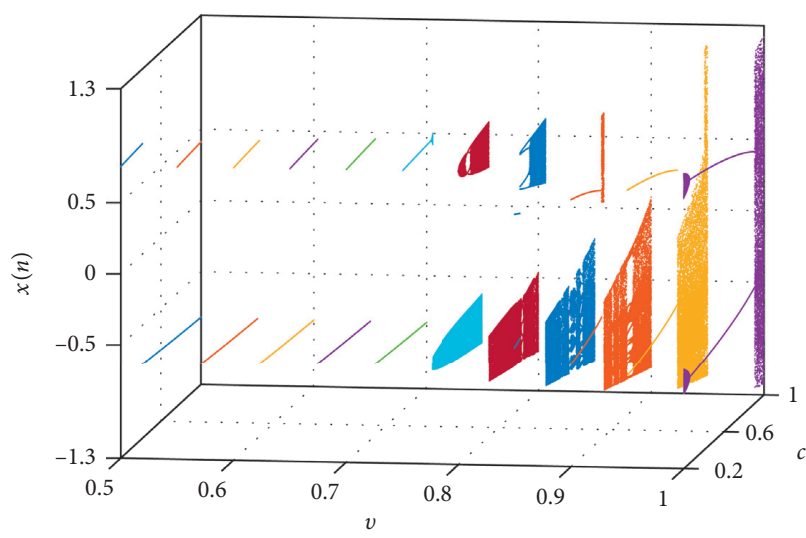

(a)

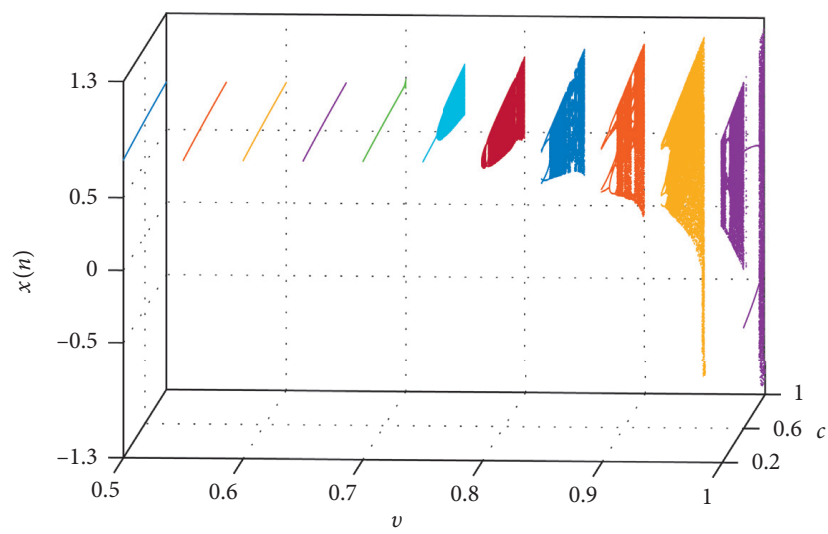

(b)

FIGURE 12: Bifurcation diagrams for map (10) with different initial values as the parameter $c$ and the order $v$ vary: (a) the bifurcation diagram with $x_{01}$ and (b) the bifurcation diagram with $x_{02}$.

\section{Stabilization}

The stabilization of map (10) will be studied in this section. From here, the case of the equilibrium to be at the origin is considered. For convenience, the controlled map (10) is rewritten as the following form:

$$
\left\{\begin{array}{l}
{ }^{C} \Delta_{a}^{v} x(t)=y(\omega)-x(\omega)+u_{1}(\omega), \\
{ }^{C} \Delta_{a}^{v} y(t)=b\left(-x^{3}(\omega)+x(\omega)\right)+c\left(-y^{3}(\omega)+y(\omega)\right)-y(\omega)+u_{2}(\omega),
\end{array}\right.
$$

where $\omega=t-1+v$ and $u_{1}$ and $u_{2}$ are the stabilization controllers.

Theorem 2. The fractional-order map (10) can be stabilized when the controllers are designed as the following form:

$$
\left\{\begin{array}{l}
u_{1}(t)=-y(t) \\
u_{2}(t)=-b\left(-x^{3}(t)+x(t)\right)-c\left(-y^{3}(t)+y(t)\right)
\end{array}\right.
$$

Proof. Through substituting (22) into (21), map (21) becomes

$$
\left\{\begin{array}{l}
{ }^{C} \Delta_{a}^{v} x(t)=-x(\omega), \\
{ }^{C} \Delta_{a}^{v} y(t)=-y(\omega) .
\end{array}\right.
$$

Map (23) can be rewritten in the compact form:

$$
{ }^{C} \Delta_{a}^{v}(x(t), y(t))^{\mathrm{T}}=\mathbf{A} \times(x(\omega), y(\omega))^{\mathrm{T}},
$$

where $\mathbf{A}=\left(\begin{array}{cc}-1 & 0 \\ 0 & -1\end{array}\right)$. Based on Theorem 1 , it is easy to see that the eigenvalues of $\mathbf{A}$ satisfy the conditions $\left|\arg \lambda_{i}\right|=\pi$ and $\left|\lambda_{i}\right|=2^{v}$, for $i=1,2$, which implies that the chaos of map (10) can be controlled and the zero equilibrium of (24) is globally asymptotically stable.

In the numerical simulations, the values of the parameters are fixed as $b=2.2$ and $c=0.95$, and the fractional order $v=0.98$. The controllers are used to stabilize map (10) when the iterations are chosen as $n=1000$. The stabilization results are displayed in Figure 13. It is clear that the state variables $x(n)$ and $y(n)$ toward to zeros, which means the chaos of map (10) is stabilized and the results confirm the theoretical control results presented in Theorem 2 .

\section{Synchronization}

Now, we consider the synchronization of map (10). Firstly, a fractional Lorenz map is taken as the drive system:

$$
\left\{\begin{array}{l}
{ }^{C} \Delta_{a}^{v} x_{d}(t)=\gamma \delta x_{d}(\omega)-\delta y_{d}(\omega) x_{d}(\omega), \\
{ }^{C} \Delta_{a}^{v} y_{d}(t)=\delta\left(-y_{d}(\omega)+x_{d}^{2}(\omega)\right)
\end{array}\right.
$$

where $0<v<1$ and state subscript $d$ denotes the drive system. Map (25) is chaotic when the parameters $\gamma=1.25$ and $\delta=0.75$ and the derivative order $v=0.98$. For more details about the dynamics of map (25), please refer to the literature [23]. Map (10) with synchronization controllers $u_{1}(\omega)$ and $u_{2}(\omega)$, which can be described by the following equations:

$$
\left\{\begin{array}{l}
{ }^{C} \Delta_{a}^{v} x_{r}(t)=y_{r}(\omega)-x_{r}(\omega)+u_{1}(\omega) \\
{ }^{C} \Delta_{a}^{v} y_{r}(t)=b\left(-x_{r}^{3}(\omega)+x_{r}(\omega)\right)+c\left(-y_{r}^{3}(\omega)+y_{r}(\omega)\right)-y_{r}(\omega)+u_{2}(\omega)
\end{array}\right.
$$

where the subscript $r$ denotes the response system. The error state variables are defined as $e_{x}(t)=x_{r}(t)-x_{d}(t)$ and $e_{y}(t)=y_{r}(t)-y_{d}(t)$. If all the error states variables tend to 0 as the time $t \longrightarrow \infty$, then maps (25) and (26) are 


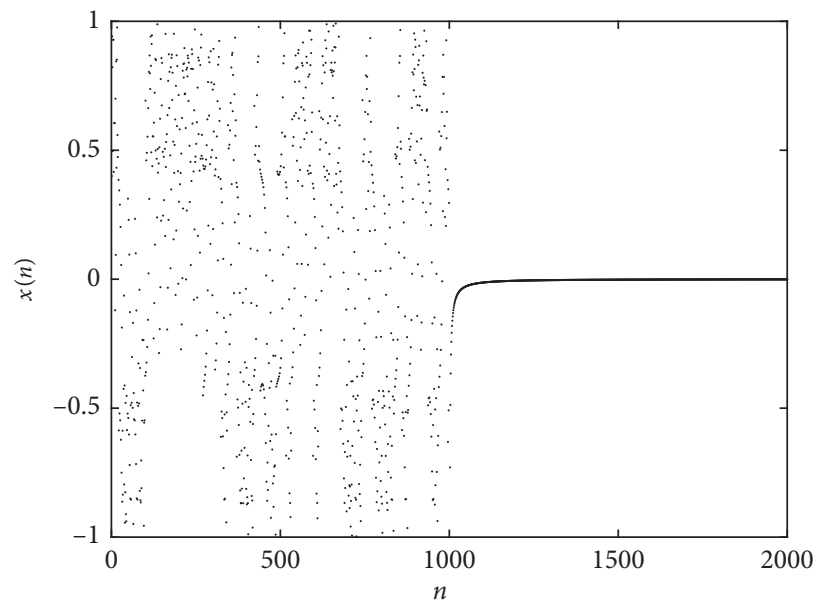

(a)

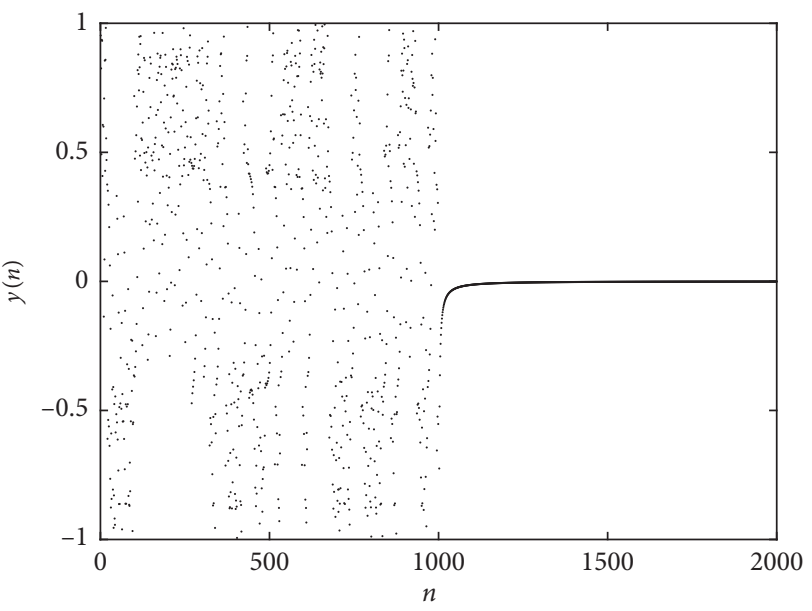

(b)

FIGURE 13: The results of the stabilization of system (10): (a) the variation of $x(n)$ with $n$ and (b) the variation of $y(n)$ with $n$.

synchronized. The following theorem is given to ensure the synchronization between the two maps can be realized.
Theorem 3. The drive and response maps (25) and (26) are synchronized when the controllers are designed as follows:

$$
\left\{\begin{array}{l}
u_{1}(\omega)=\gamma \delta x_{d}(\omega)-\delta y_{d}(\omega) x_{d}(\omega)-y_{d}(\omega)+x_{d}(\omega), \\
u_{2}(\omega)=\delta\left(-y_{d}(\omega)+x_{d}^{2}(\omega)\right)-b\left(-x_{r}^{3}(\omega)+x_{r}(\omega)\right)-c\left(-y_{r}^{3}(\omega)+y_{r}(\omega)\right)+y_{d}(\omega)
\end{array}\right.
$$

Proof. The error dynamical system with fractional Caputo difference is

$$
\left\{\begin{array}{l}
{ }^{C} \Delta_{a}^{v} e_{1}(t)=y_{r}(\omega)-x_{r}(\omega)-\gamma \delta x_{d}(\omega)+\delta y_{d}(\omega) x_{d}(\omega)+u_{1}(\omega), \\
{ }^{C} \Delta_{a}^{v} e_{2}(t)=b\left(-x_{r}^{3}(\omega)+x_{r}(\omega)\right)+c\left(-y_{r}^{3}(\omega)+y_{r}(\omega)\right)-y_{r}(\omega)-\delta\left(-y_{d}(\omega)+x_{d}^{2}(\omega)\right)+u_{2}(\omega)
\end{array}\right.
$$

By substituting controllers (27) into (28), we can obtain the error dynamical system:

$$
\left\{\begin{array}{l}
{ }^{C} \Delta_{a}^{v} e_{1}(t)=e_{2}(\omega)-e_{1}(\omega) \\
{ }^{C} \Delta_{a}^{v} e_{2}(t)=-e_{2}(\omega)
\end{array}\right.
$$

System (29) is rewritten in the compact form:

$$
{ }^{\mathrm{C}} \Delta_{a}^{v}\left(e_{1}(t), e_{2}(t)\right)=\mathbf{M} \times\left(e_{1}(\omega), e_{2}(\omega)\right)^{\mathrm{T}},
$$

where $\mathbf{M}=\left[\begin{array}{cc}-1 & 1 \\ 0 & -1\end{array}\right]$. It can be seen that the eigenvalues of the matrix $\mathbf{M}$ satisfy the following stability condition:

$$
\begin{aligned}
\left|\lambda_{i}\right| & <\left(2 \cos \frac{\left|\arg \lambda_{i}\right|-\pi}{2-v}\right)^{v}, \\
\left|\arg \lambda_{i}\right| & >\frac{v \pi}{2}, \quad i=1,2 .
\end{aligned}
$$

Based on Theorem 1, we can get that the zero equilibrium of (29) is globally asymptotically stable, which implies the two maps (25) and (26) are synchronized.

In the numerical simulations, the parameters are fixed as $\gamma=1.25, \delta=0.75, b=2.2$, and $c=0.95$ and the order is $v=0.98$. The initial conditions of the two systems (25) and (26) are $\left(x_{d 0}, y_{d 0}\right)=(0.1,0.1)$ and $\left(x_{r 0}, y_{r 0}\right)=(0.8,0.4)$. 


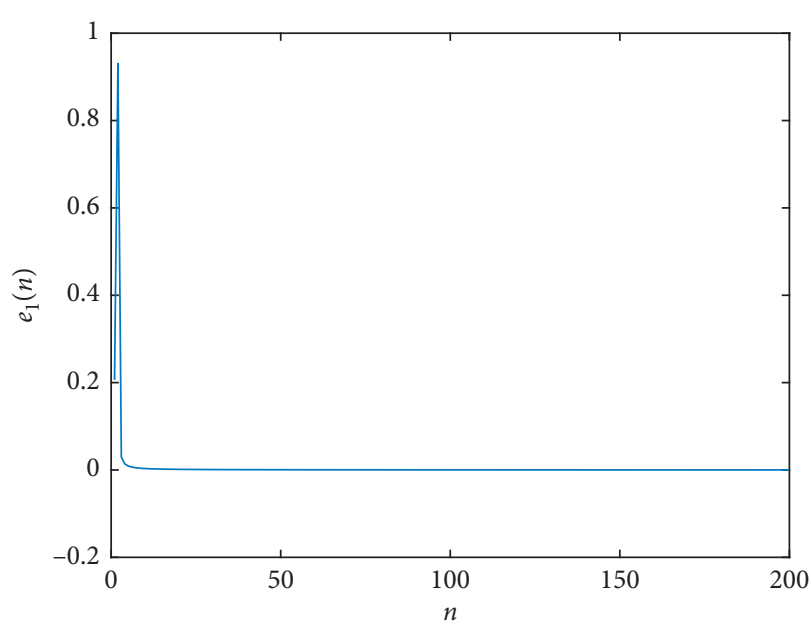

(a)

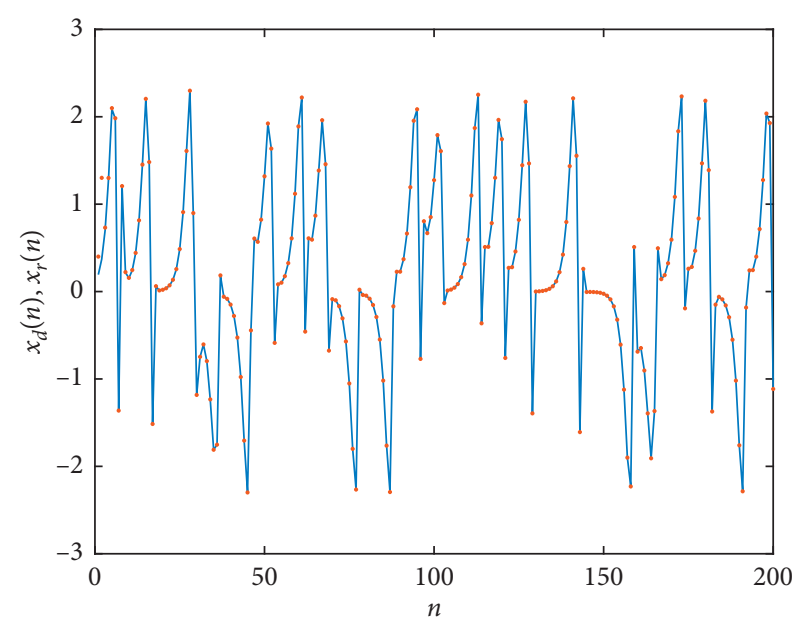

(c)

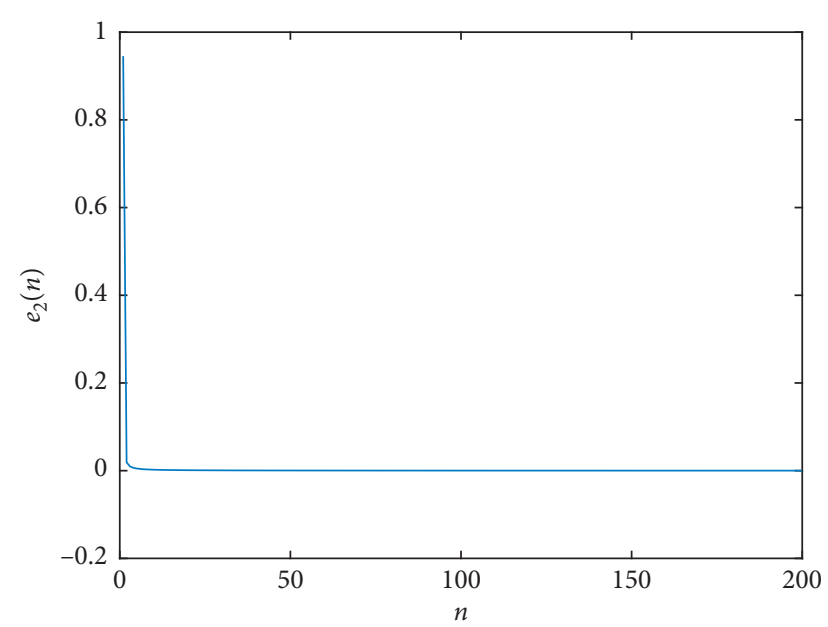

(b)

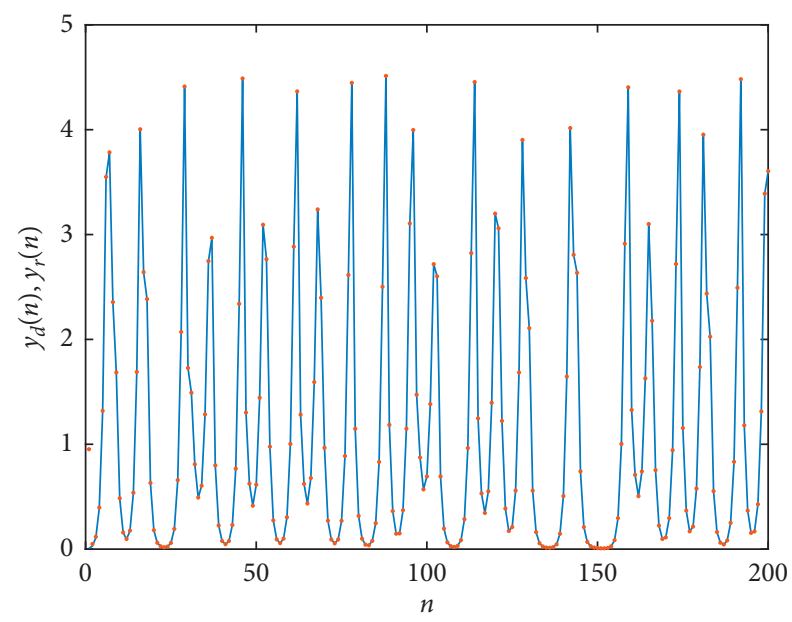

(d)

FIGURE 14: The simulation results for the synchronization of map (10): (a) the error state variable $e_{1}(n)$ with the variation of $n$, (b) the error state variable $e_{2}(n)$ with the variation of $n,(\mathrm{c})$ the state variables $x_{d}(n)$ and $x_{r}(n)$ with the variation $n$, and (d) the state variables $y_{d}(n)$ and $y_{r}(n)$ with the variation $n$.

The results of numerical simulations are depicted in Figure 14. From which, we can see that the error variables $e_{1}$ and $e_{2}$ converge to zero rapidly as $n$ increases (Figures 14(a) and 11(b)). Meanwhile, the evolution of the state variables with time $n$ for the two maps (25) and (26) are synchronized under the designed controllers (27) (Figures 14(c) and 14(d)).

\section{Conclusions and Discussion}

A fractional-order discrete noninvertible map with cubic nonlinearity is proposed in this paper. Firstly, the stability of the equilibrium points for the map is analyzed. Secondly, the dynamics of the map with two different initial conditions is studied by numerical simulation. Bifurcation diagrams and phase plots are obtained as a parameter or the fractional order varies. A series of attractors of the map in different forms, including equilibrium points, limit cycles, and chaotic attractors, are plotted. Furthermore, bifurcations with the simultaneous variation of both parameter and order are also analyzed in the three-dimensional space. From the global dynamics perspective, interior crises occur in the map as a parameter or the order varies. Thirdly, based on the stability theory of fractional-order discrete maps, the chaos of the map is controlled by the stabilization controllers. Finally, the synchronization between the proposed map and a fractional-order discrete Loren map is investigated. Numerical simulations are implemented to verify the effectiveness of the designed controllers. The results obtained in this paper reveal that chaos really exists in the fractional-order formation for the map proposed in [31-33]. More abundant local and global dynamics are found in the fractional-order map.

It is worth mentioning that the mechanism of interior crises occur in map (10) cannot be displayed from a global perspective due to the absence of effective global dynamics computation methods for fractional-order discrete maps. Therefore, developing the effective computation methods of global analysis for this kind of systems is our following work. 


\section{Data Availability}

The data for the bifurcation diagrams used to support the findings of this study are included within the supplementary information files (available here). For the large of the data, we will supply the bifurcation data calculated by the software Matlab.

\section{Conflicts of Interest}

The authors declare that they have no conflicts of interest.

\section{Acknowledgments}

This work was supported by the National Natural Science Foundation of China (nos. 11702194 and 11702195) and Natural Science Preparatory Study Foundation of Xi'an University of Posts and Telecommunications (no. 106/ 205020030).

\section{Supplementary Materials}

(1) discretesanweiIN1q098b and discretesanweiIN2q098b: the data of Figures 1(a) and 1(b). (2) discretesanweiIN1q098c and discretesanweiIN2q098c: the data of Figures 4(a) and 4(b). (3) discretesanweiqIN1 and discretesanweiqIN2: the data of Figures 8(a) and 8(b). (Supplementary Materials)

\section{References}

[1] R. M. May, "Simple mathematical models with very complicated dynamics," Nature, vol. 261, no. 21, pp. 459-467, 1976.

[2] H. Sakaguchi and K. Tomita, "Bifurcations of the coupled Logistic map," Progress of Theoretical Physics, vol. 78, no. 2, pp. 305-315, 1987.

[3] M. J. Feigenbaum, "Quantitative universality for a class of nonlinear transformations," Journal of Statistical Physics, vol. 19, no. 1, pp. 25-52, 1978.

[4] M. Hénon, "A two-dimensional mapping with a strange attractor," Communications in Mathematical Physics, vol. 50, no. 1, pp. 69-77, 1976.

[5] P. R. Lozi, "Un atracteur étrange du type attracteur de Hénon," Journal de Physique, vol. 39, pp. 9-10, 1978.

[6] C. P. Li, W. H. Deng, and D. Xu, "Chaos synchronization of the Chua system with a fractional order," Physica A: Statistical Mechanics and Its Applications, vol. 360, no. 2, pp. 171-185, 2006.

[7] J. G. Lu and G. Chen, "A note on the fractional-order Chen system," Chaos, Solitons \& Fractals, vol. 27, no. 3, pp. 685-688, 2006.

[8] C. Zeng, Q. Yang, and J. Wang, "Chaos and mixed synchronization of a new fractional-order system with one saddle and two stable node-foci," Nonlinear Dynamics, vol. 65, no. 4, pp. 457-466, 2011.

[9] R. Behinfaraz and M. A. Badamchizadeh, "Synchronization of different fractional order chaotic systems with time-varying parameter and orders," ISA Transactions, vol. 80, pp. 399-410, 2018.

[10] D. Cafagna and G. Grassi, "Bifurcation and chaos in the fractional-order Chen system via a time-domain approach,"
International Journal of Bifurcation and Chaos, vol. 18, no. 7, pp. 1845-1863, 2008.

[11] J. Huo, H. Zhao, and L. Zhu, "The effect of vaccines on backward bifurcation in a fractional order HIV model," Nonlinear Analysis: Real World Applications, vol. 26, pp. 289-305, 2015.

[12] X. Li and R. Wu, "Hopf bifurcation analysis of a new commensurate fractional-order hyperchaotic system," Nonlinear Dynamics, vol. 78, no. 1, pp. 279-288, 2014.

[13] A. Y. T. Leung, H. X. Yang, and P. Zhu, "Bifurcation of a Duffing oscillator having nonlinear fractional derivative feedback," International Journal of Bifurcation and Chaos, vol. 24, no. 3, Article ID 1450028, 2014.

[14] A. M. A. El-Sayed, A. Elsonbaty, A. A. Elsadany, and A. E. Matouk, "Dynamical analysis and circuit simulation of a new fractional-order hyperchaotic system and its discretization," International Journal of Bifurcation and Chaos, vol. 26, no. 13, Article ID 1650222, 2016.

[15] A. E. Matouk and A. A. Elsadany, "Dynamical analysis, stabilization and discretization of a chaotic fractional-order GLV model," Nonlinear Dynamics, vol. 85, no. 3, pp. 15971612, 2016.

[16] X. Rui, W. Yin, Y. Dong, L. Lin, and X. Wu, "Fractional-order sliding mode control for hybrid drive wind power generation system with disturbances in the grid," Wind Energy, vol. 22, no. 1, pp. 49-64, 2019.

[17] R. Behinfaraz, S. Ghaemi, and S. Khanmohammadi, "Adaptive synchronization of new fractional order chaotic systems with fractional adaption laws based on risk analysis," Mathematical Methods in the Applied Sciences, vol. 42, no. 6, pp. 1772-1785, 2019.

[18] R. Behinfaraz, S. Ghaemi, and S. Khanmohammadi, "Risk assessment in control of fractional-order coronary artery system in the presence of external disturbance with different proposed controllers," Applied Soft Computing, vol. 77, pp. 290-299, 2019.

[19] C. Goodrich and A. C. Peterson, Discrete Fractional Calculus, Springer, New York, NY, USA, 2015.

[20] J. B. Diaz and T. J. Osler, "Differences of fractional order," Mathematics of Computation, vol. 28, no. 125, pp. 185-202, 1974.

[21] Y. Liu, "Discrete chaos in fractional Hénon maps," International Journal of Nonlinear Science, vol. 18, no. 3, pp. 170-175, 2014.

[22] A. Ouannas, A.-A. Khennaoui, Z. Odibat, V.-T. Pham, and G. Grassi, "On the dynamics, control and synchronization of fractional-order Ikeda map," Chaos, Solitons \& Fractals, vol. 123, pp. 108-115, 2019.

[23] A. A. Khennaoul, A. Ouannas, S. Bendoukha, G. Grassi, P. R. Lozi, and V. T. Pham, "On fractional-order discrete-time systems: chaos, stabilization and synchronization," Chaos, Solitons and Fractals, vol. 119, pp. 150-162, 2019.

[24] J. Mumkhamar, "Chaos in a fractional order logistic map," Fractional Calculus and Applied Analysis, vol. 16, no. 3, pp. 511-519, 2013.

[25] G. C. Wu, D. Baleanu, and S. D. Zeng, "Discrete chaos in fractional sine and standard maps," Physics Letters A, vol. 378, no. 5-6, pp. 484-487, 2014.

[26] A. A. Khennaoul, A. Ouannas, S. Bendoukha, G. Grassi, $\mathrm{X}$. Wang, and V. T. Pham, "Generalized and inverse generalized synchronization of fractional-order discrete-time chaotic systems with non-identical dimension," Advances Difference Equations, vol. 2018, Article ID 303, 2018. 
[27] O. Megherbi, H. Hamiche, S. Djennoune, and M. Bettayeb, "A new contribution for the impulsive synchronization of fractional-order discrete-time chaotic systems," Nonlinear Dynamics, vol. 90, no. 3, pp. 1519-1533, 2017.

[28] Y. Ji, L. Lai, S. Zhong, and L. Zhang, "Bifurcation and chaos of a new discrete fractional-order logistic map," Communications in Nonlinear Science and Numerical Simulation, vol. 57, pp. 352-358, 2018.

[29] H. Sun, Y. Zhang, D. Baleanu, W. Chen, and Y. Chen, "A new collection of real world applications of fractional calculus in science and engineering," Communications in Nonlinear Science and Numerical Simulation, vol. 64, pp. 213-231, 2018.

[30] G.-C. Wu and D. Baleanu, "Discrete chaos in fractional delayed logistic maps," Nonlinear Dynamics, vol. 80, no. 4, pp. 1697-1703, 2015.

[31] C. Mira, D. Fournier-Prunaret, L. Gardini, H. Kawakami, and J. C. Cathala, "Basin bifurcations of two-dimensional noninvertible maps: fractalization of basins," International Journal of Bifurcation and Chaos, vol. 4, no. 2, pp. 342-382, 1994.

[32] D. Fourier-Prunaret and V. Guglielmi, "Bifurcations and attractors in two-dimensional maps of cubic type," in Proceedings of the international conference on nonlinear theory and applications 1999, Hawaï, USA, November-December 1999.

[33] V. Guglielmi, H. Poonith, D. Fourier-Prunaret, and A. K. Taha, "Security performances of a chaotic cryptosystem," in Proceedings of the 2004 IEEE International Symposium on Industrial Electronics, vol. 1, Ajaccio, France, May 2004.

[34] T. Abdeljawad, "On Riemann and Caputo fractional differences," Computers \& Mathematics with Applications, vol. 62, no. 3, pp. 1602-1611, 2011.

[35] H. L. Gray and N. F. Zhang, "On a new definition of the fractional difference," Mathematics of Computation, vol. 50, no. 182 , p. $513,1988$.

[36] K. S. Miller and B. Ross, Univalent Functions, Fractional Calculus, and Their Applications, Eills Howard, Chichester, UK, 1989.

[37] J. Čermák, I. Györi, and L. Něchvátal, “On explicit stability condition for a linear fractional difference system," Fractional Calculus and Applied Analysis, vol. 18, no. 3, pp. 651-672, 2015.

[38] D. Baleanu, G. C. Wu, Y. R. Bai, and F. L. Chen, "Stability analysis of Caputo-like discrete fractional systems," Communications in Nonlinear Science and Numerical Simulation, vol. 48, pp. 520-530, 2017. 